\title{
The Far Ultra-Violet Imager on the Icon Mission
}

\author{
S.B. Mende ${ }^{1} \cdot$ H.U. Frey ${ }^{1}$ - K. Rider ${ }^{1}$. C. Chou $^{1}$ - S.E. Harris ${ }^{1}$ - O.H.W. Siegmund ${ }^{1}$. \\ S.L. England ${ }^{1}$ - C. Wilkins ${ }^{1}$ - W. Craig1 • T.J. Immel ${ }^{1}$ P. Turin ${ }^{1}$ - N. Darling ${ }^{1}$.

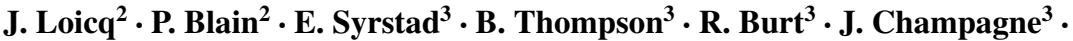 \\ P. Sevilla ${ }^{3} \cdot$ S. Ellis ${ }^{4}$
}

Received: 18 December 2016 / Accepted: 24 May 2017

(C) Springer Science+Business Media B.V. 2017

\begin{abstract}
ICON Far UltraViolet (FUV) imager contributes to the ICON science objectives by providing remote sensing measurements of the daytime and nighttime atmosphere/ionosphere. During sunlit atmospheric conditions, ICON FUV images the limb altitude profile in the shortwave (SW) band at $135.6 \mathrm{~nm}$ and the longwave (LW) band at 157 $\mathrm{nm}$ perpendicular to the satellite motion to retrieve the atmospheric $\mathrm{O} / \mathrm{N}_{2}$ ratio. In conditions of atmospheric darkness, ICON FUV measures the $135.6 \mathrm{~nm}$ recombination emission of $\mathrm{O}^{+}$ions used to compute the nighttime ionospheric altitude distribution. ICON Far UltraViolet (FUV) imager is a Czerny-Turner design Spectrographic Imager with two exit slits and corresponding back imager cameras that produce two independent images in separate wavelength bands on two detectors. All observations will be processed as limb altitude profiles. In addition, the ionospheric $135.6 \mathrm{~nm}$ data will be processed as longitude and latitude spatial maps to obtain images of ion distributions around regions of equatorial spread $\mathrm{F}$. The ICON FUV optic axis is pointed 20 degrees below local horizontal and has a steering mirror that allows the field of view to be steered up to 30 degrees forward and aft, to keep the local magnetic meridian in the field of view. The detectors are micro channel plate (MCP) intensified FUV tubes with the phosphor fiber-optically coupled to Charge Coupled Devices (CCDs). The dual stack MCP-s amplify the photoelectron signals to overcome the CCD noise and the rapidly scanned frames are co-added to digitally create 12-second integrated images. Digital on-board signal processing is used to compensate for geometric distortion and satellite motion and to achieve data compression. The instrument was originally aligned in visible light by using a special grating and visible cameras. Final alignment, functional and environmental testing and calibration were performed in a large vacuum chamber with
\end{abstract}

The Ionospheric Connection Explorer (ICON) mission

Edited by Doug Rowland and Thomas J. Immel

\footnotetext{
S.B. Mende

mende@ssl.berkeley.edu

1 University of California Berkeley, Berkeley, USA

2 Centre Spatial de Liege (CSL), Liege, Belgium

3 Space Dynamics Lab., Utah State University, Logan, USA

4 Photon Engineering, Tucson, USA
}

Published online: 01 August 2017 
a UV source. The test and calibration program showed that ICON FUV meets its design requirements and is ready to be launched on the ICON spacecraft.

Keywords Space-borne far ultraviolet spectral imager · Imaging the Earth's atmosphere/ionosphere $\cdot$ Instrument for observing the upper atmosphere

\section{Introduction}

Recent efforts to understand the coupling across atmospheric regions show that the interaction between neutral particles and ions involves a set of complex electrodynamic processes. Unraveling the details behind these interactions requires a science mission. The Ionospheric Connection Explorer (ICON) is dedicated to making continuous observations of the neutral atmospheric drivers and the resulting ionospheric responses (Immel et al. 2017). The thermospheric winds, densities, temperatures, and composition are measured in the region 100-400 kilometers above the Earth as well as the related conditions in the ionosphere. An instrument operating in the Far Ultra Violet (FUV) spectral region (100-200 nm) is capable of measuring emissions that characterize several important components of the atmosphere/ionosphere i.e. the thermosphere system.

The ICON FUV instrument was designed to make measurements of the daytime atomic oxygen $\mathrm{O}$ to molecular nitrogen $\mathrm{N}_{2}$ composition ratio of the atmosphere because it is a significant driver of the system. The same instrument will also be able to make measurements of the nighttime ionospheric density altitude distribution, which is a significant response of the ionosphere to all drivers.

In this paper we describe the FUV imager on board the ICON S/C. First we present the scientific justifications for flying such an instrument on the ICON mission. This is followed by a discussion of the detailed scientific requirements and a requirement summary including the required responsivity of the ICON FUV instrument. In the next section we define the instrument responsivity for a generalized imaging instrument and show how it depends on the chosen properties of the instrument. A description of the instrument operating principle is given explaining the difference between a Spectrographic Imager like ICON FUV and other FUV spectrometers like GUVI or GOLD. A brief overview of the ICON FUV imager instrument is given including a section that explains how the data products are obtained and why there is a requirement for extensive on board data processing. The next section provides the overall philosophy of how the instrument was developed including the pre-alignment of the reflective parts of the instrument using visible light to validate the basic design and to permit the instrument operation and alignment in laboratory air prior to the instrument final alignment using FUV light in the vacuum chamber. This is followed by the detailed presentation of the instrument optical design and performance. We compared the predicted design performance to the actually realized calibrated performance in wavelength, spatial resolutions, geometric distortion and stray light rejection. The special high sensitivity and high dynamic range FUV detectors are presented in the next section. Since the ICON FUV detectors do not produce digital photoelectron counts but digitized numbers proportional to the analog charge in the output CCD-s, we discuss how these signal can be converted to equivalent output photoelectron counts that allow the estimation of the ICON FUV responsivity. In the last section we summarize the calibration results in Tables 7 and 8 and compare the ICON FUV predicted responsivities to a similar FUV instrument. 


\section{ICON FUV Monitoring of Atmospheric and Ionospheric Constituents}

On the dayside there are key emissions of molecular $\mathrm{N}_{2}$ and atomic $\mathrm{O}$ excited by photo electron impact. Atomic $\mathrm{O}$ emission are also created by recombination of oxygen ions (Fig. 1), which is the dominant sources on the nightside. The $\mathrm{N}_{2}$ emissions in the Lyman-BirgeHopfield ( $\mathrm{LBH}$ ) bands are found in a broad range of wavelengths from $125 \mathrm{~nm}$ to longer than $200 \mathrm{~nm}$, mixed with several single atomic lines of $\mathrm{N}$ and $\mathrm{O}$. The ratio of $\mathrm{N}_{2}$ and $\mathrm{O}$ emission intensities depends on the density ratio. In Fig. 1 on the top right we illustrate schematically how viewing the atmosphere from the limb from a Space Craft (S/C) allows the inversion of the limb emission profiles into volume emission rates as a function of altitude. It should be noted that the method assumes that the emission intensity at a particular altitude is constant over a relatively large region around the measurement.

There is minimal excitation of these emission features on the nightside, but the ionized $\mathrm{O}^{+}$takes a long time to recombine and the intensity of the emission caused by the nightside residual oxygen ions $\left(\mathrm{O}^{+}\right)$recombining with thermal electrons provides a good measure of the nightside $\mathrm{O}^{+}$ion density.

\subsection{Space Based Observations of the Atmosphere in the FUV}

Previously flown satellite imaging experiments have demonstrated the suitability of the vacuum ultraviolet spectral region for observing the upper atmosphere (Anger et al. 1987; Frank et al. 1981; Frank and Craven 1988; Murphree et al. 1994; Torr et al. 1995, Mende et al. 2000a, 2000b, 2000c; Christensen et al. 2003). The primary focus of the prior imaging experiments was to study the high latitude regions mainly the aurora. One of the main advantages of observing the atmosphere in the FUV is that the atmosphere is opaque below the $\mathrm{O}_{2}$ layer $(<130 \mathrm{~km})$ and in the wavelength region $120-190 \mathrm{~nm}$ a downward-viewing imager is minimally contaminated by scattered sunlight from clouds and the ground.

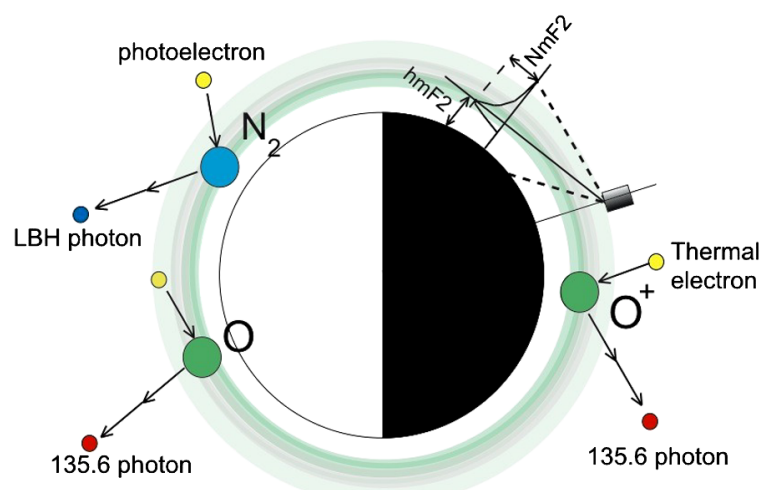

Fig. 1 On the dayside, ICON measures limb profiles of $\mathrm{N}_{2}$ and atomic $\mathrm{O}$ emissions excited by photo electron impact, to obtain the ratio of the densities of $\mathrm{N}_{2}$ and $\mathrm{O}$. On the nightside it measures the limb profile of the nightside $\mathrm{O}^{+}$emission. From this the recombination rate and the dominant $\mathrm{O}^{+}$ion density altitude distribution may be deduced. The relationship between limb emission intensity and volume emission rate is illustrated on the top right illustrating how the ionospheric F2 layer peak density (NmF2) and peak altitude (hmF2) are obtained 


\subsubsection{Thermospheric Neutral Species}

Meier and Anderson (1983) proposed the measurement of unique spectral signatures of the number densities and temperature of the major thermospheric neutral species $\left(\mathrm{N}_{2}, \mathrm{O}_{2}\right.$, and O) in the FUV. Meier and Picone (1994) showed that limb scans of FUV emissions can be inverted to yield altitude profiles of neutral concentrations and thus established the value of utilizing FUV emissions observed from low Earth-orbiting satellites to systematically monitor thermospheric composition and temperature as functions of altitude, geographic location, and time. This motivated the inclusion of the Global Ultraviolet Imager (GUVI), an FUV spectro-radiometer, on the Thermosphere Ionosphere Mesosphere Energetics and Dynamics (TIMED) satellite, launched in 2001 (Christensen et al. 2003). GUVI provided FUV radiances measured on the Earth limb which were transformed into O, N2, and O2 number densities and temperature (Meier et al. 2015), thus providing data regarding composition and temperature variations between 2002 and 2007. Techniques were developed for GUVI limb data products for the retrieval of thermospheric neutral composition and temperature using forward modeling (e.g. Meier et al. 2015). These techniques are applicable to ICON FUV. In addition to atmospheric composition and temperature GUVI data was also used to infer the geo-effective EUV solar irradiance (Strickland et al. 1995), which was compared to simultaneous Solar Extreme EUV (SEE) measurements on TIMED. It appears that the EUV flux estimated from GUVI measurement was on average 30\% lower than the SEE measurement and varied less with solar activity. Although the interpretation of the geoeffective EUV solar irradiance is not listed as a required ICON data product, it could be a significant added benefit for modeling, especially for E-region conductivity.

\subsubsection{Nighttime Ionosphere}

Imaging of OI $135.6 \mathrm{~nm}$ FUV light from a satellite is a powerful tool for investigating global characteristics of the earth's ionosphere. OI $135.6 \mathrm{~nm}$ emission in the nighttime ionosphere is produced mostly by radiative recombination of the atomic oxygen ion. Another source of $135.6 \mathrm{~nm}$ is ion-ion neutralization (Hanson 1970) but can be considered a minor source except after midnight (Qin et al. 2016). The $135.6 \mathrm{~nm}$ emission intensity is approximately proportional to the recombination rate and therefore to the product of $\mathrm{O}^{+}$density and of the electron density. In the altitude region of interest the $\mathrm{O}^{+}$ions are dominant and $\mathrm{O}^{+}$density squared is a good approximation for the product of $\mathrm{O}^{+}$and of the electron density. Thus the intensity of the $135.6 \mathrm{~nm}$ emission can be used to obtain the $\mathrm{O}^{+}$ion density.

The OI $135.6 \mathrm{~nm}$ emission from the low latitude ionosphere was first observed by the OGO-4 satellite, and it was observed that the low-latitude ionosphere had two emission peaks located $10^{\circ}-20^{\circ}$ from the geomagnetic equator corresponding to peaks of the plasma density of the equatorial anomaly (EA) or a.k.a. Appleton anomaly (Hicks and Chubb 1970; Barth and Schaffner 1970). Huba et al. (2002) reported that the $\mathrm{O}^{+}$density deduced from the OI $135.6 \mathrm{~nm}$ limb scan data agrees well with the SAMI2 ionosphere model. The NASA IMAGE mission had three FUV cameras for imaging the upper atmosphere at three FUV wavelength regions, one with the pass band centered at $135.6 \mathrm{~nm}$ and with an 8-nm bandwidth (Mende et al. 2000c). This SI-13 channel was sensitive enough to image the lowlatitude ionosphere at night. Several global features of the low latitude ionosphere were observed by the IMAGE FUV experiment (Sagawa et al. 2003). The dynamics of the low latitude EA were investigated by Immel et al. (2006) and systematic longitudinal variations in ionospheric density were discovered with $1000-\mathrm{km}$ scale length. They were associated with the strength of non-migrating, diurnal atmospheric tides that are, in turn, driven mainly 
by weather in the tropics. It was considered surprising that non-migrating tides could influence the ionosphere. It showed a strong connection between tropospheric and ionospheric conditions, in spite of our expectation that the upward propagating tides are damped out far below the F region peak of ionospheric density. ICON carries this investigation forward with highly sensitive measurements in this same wavelength range, now with limb imaging and a steerable field of view.

\subsubsection{Equatorial Spread F}

The nighttime equatorial ionosphere is highly unstable with plasma turbulence occurring regularly causing radio wave scintillations by density irregularities (commonly referred to as "Equatorial Spread F", or ESF). These irregularities are responsible for outages of communication and navigation systems using trans-ionospheric radio links. These ESF events are produced by plasma density depletions originating in the post-sunset bottom side ionosphere. These depletions or plasma bubbles are longitudinally narrow but highly extended along magnetic field lines and optically detectable by imaging (Makela et al. 2001). Ionospheric plasma bubbles seem to occur on one day but are completely absent on another seemingly under identical ionospheric conditions (Kamalabadi et al. 2009). These bubbles were studied in the far ultraviolet (Comberiate et al. 2007) using the GUVI instrument on TIMED. The ICON FUV instrument provides a new opportunity to image and study the bubbles and the associated ionospheric conditions.

\subsection{Detailed Scientific Requirements for ICON FUV Observations}

The spectrum of the dayglow of the upper atmosphere is illustrated in Fig. 2. Lines of the LBH band system are illustrated in black while the $135.6 \mathrm{~nm} \mathrm{O}$ emission is in green. ICON FUV was designed to pick up parts of the $\mathrm{LBH}$ bands to measure the daytime $\mathrm{N}_{2}$ component of atmospheric density while the $\mathrm{O}$ component is measured via the $135.6 \mathrm{O}$ atomic line. The same 135.6 line is used at night for the ionospheric ion density measurement. Three

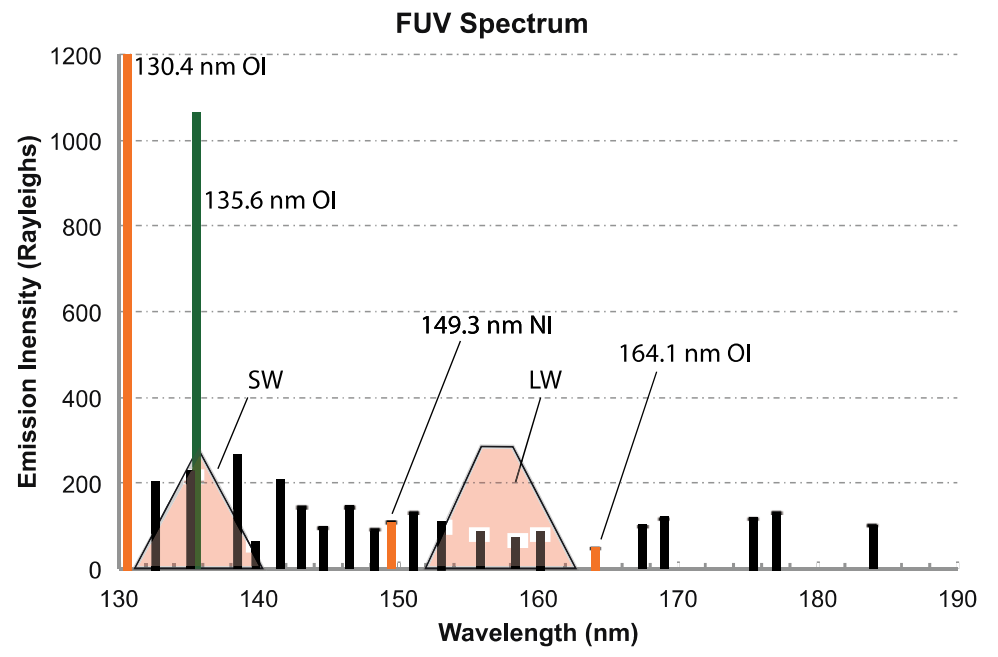

Fig. 2 FUV dayglow spectrum. LBH band (black), OI $135.6 \mathrm{~nm}$ (green), and undesirable OI and NI lines in orange (Color figure online) 

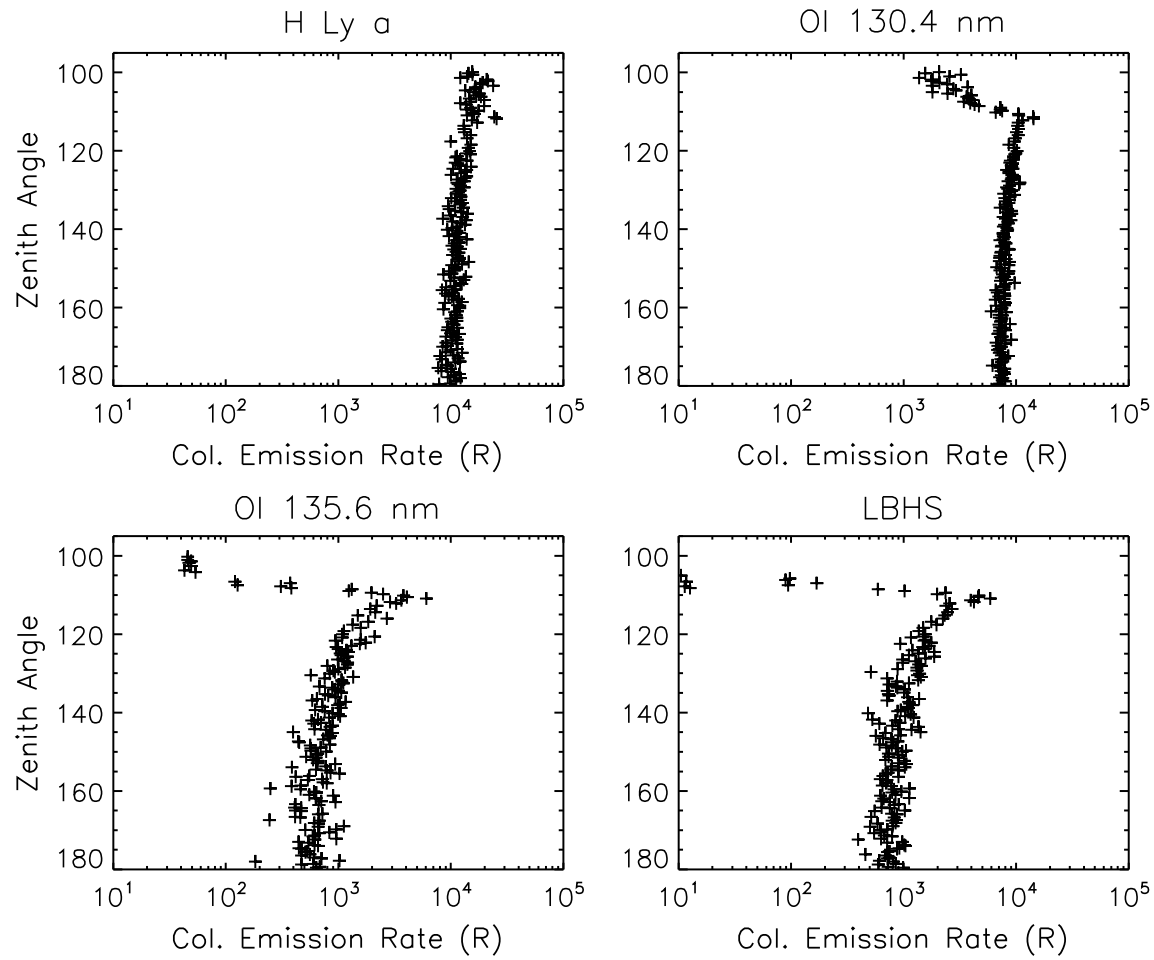

Fig. 3 Limb Radiance profiles showing Lyman alpha (121.6 nm), $130.4 \mathrm{~nm}, 135.6 \mathrm{~nm}$ and LBHS covering a range of LBH lines from GUVI taken on TIMED (Rev 13505) at $612 \mathrm{~km}$ altitude on day 158 of 2004 (Meier 2017 , private communication)

undesirable emission features are shown in orange color and they represent the optically thick $130.4 \mathrm{~nm} \mathrm{O}$ line, the $149.3 \mathrm{~nm} \mathrm{~N}$ line, and the $164.1 \mathrm{~nm} \mathrm{O}$ line. Including these lines into the FUV instrumental passband would make the data analysis more difficult and it was a specific requirement to avoid these features. ICON FUV spectral regions are illustrated on Fig. 2 and labeled as Long Wave (LW) and Short Wave (SW) bands. The requirement of transmitting the $135.6 \mathrm{~nm}$ line and rejecting the 130.4 dictated the most stringent spectral resolution requirement.

Limb Radiance profiles on the dayside are shown in Fig. 3.

Lyman alpha $(121.6 \mathrm{~nm})$ is the brightest emission reaching almost several tens of $\mathrm{kR}$ near the limb. Suppressing Lyman alpha therefore is of paramount importance. The same goes for the $130.4 \mathrm{~nm}$ OI feature, as it is also very bright, and being optically thick cannot be used for direct inversion of the limb emission profiles. We show an LBH sample from GUVI known as LBHS covering a range of LBH lines (Meier 2017, private communication).

A top view of the ICON FUV observing scenario is shown in Fig. 4 with three positions of the S/C along the orbit track. First from position (1) and then about seven minutes later from position (3) the fore- and aft-viewing ICON Michelson interferometer (MIGHTI-Fore and MIGHTI-Aft) measure the Doppler shift of the line of sight components of the airglow emitted by the neutral wind driven atmosphere. From the two Doppler measurements the actual vector direction and magnitude of the wind can be derived at locations labeled as "tangent height track". It is an interesting property of the dipole magnetic field that near the 
Fig. 4 Top view of the ICON observing scenario with the S/C at three positions along the orbit track. The EUV fixed FOV is illustrated in blue while the horizontally steered FUV is illustrated in yellow (Color figure online)
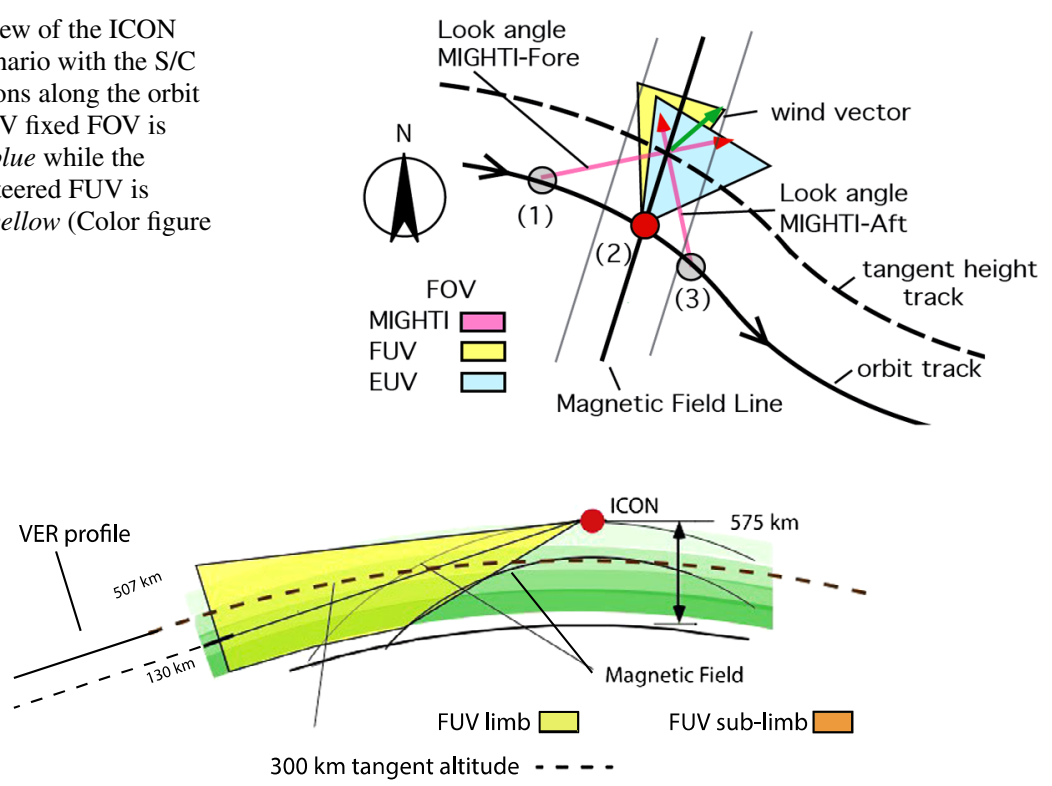

Fig. 5 Side view of ICON FUV viewing geometry (not to scale). ICON S/C is shown travelling into the page (eastward) on the magnetic equator at $575 \mathrm{~km}$ altitude with FUV viewing on the port side with the central ray observing at 20 degrees below local horizontal. Volume Emission Rate (VER) profile with altitude for nighttime $135.6 \mathrm{~nm}$ emission shown on left

magnetic equator this MIGHTI tangent height track is very close to the same as the track of points at the same altitude on the arched magnetic field line. The ICON ion drift meter measures the ion velocity at the S/C on the same field line at position (2) where the wind is also measured, therefore the measured ion drift and corresponding electric field at the spacecraft can be mapped along the magnetic field to the wind altitude profile measurements. At position (2), the FUV and the Extreme-UV (EUV) instruments take a set of limb view images of the atmosphere and ionosphere also near the footprint of ICON's magnetic field line. On Fig. 4, the EUV fixed field of view is illustrated in blue whereas the horizontally steered FUV field of view is illustrated in yellow. At night time the equatorial ionosphere is structured and the structures are aligned along the magnetic field therefore it is advantageous to observe them in a direction parallel to the magnetic field. There is a requirement that on the night side the FUV field of view is steered to be parallel with the local magnetic field at ICON.

The ICON FUV nighttime viewing geometry is illustrated as a side view on Fig. 5. The $24^{\circ}$ vertical FOV covers a region nominally from -8 to -32 degree elevation. Two arched equatorial magnetic field lines are also illustrated. At the left of the figure we show a schematic volume emission rate (VER) profile with altitude for nighttime $135.6 \mathrm{~nm}$ emission. The observed intensity is limited at the bottom by the $\mathrm{O}_{2}$ FUV extinction at about $130 \mathrm{~km}$ altitude. The figure shows the nighttime ionospheric emissions peaking around $300 \mathrm{~km}$. The $300 \mathrm{~km}$ altitude line is indicated by a dashed curve. If all the emissions were generated at this altitude then ICON FUV would view them as a two dimensional scene observed at a slant view from above in the view angle region of tangent height of $300 \mathrm{~km}$ and below, shown in orange. This viewing scenario is designated as "FUV sub-limb" region. Emissions above the $300 \mathrm{~km}$ tangent height are mapped according to their tangent 
altitude. This viewing scenario is illustrated as "FUV limb" and is shown in yellow color. Processing the two dimensional FUV imaging data and compensating for satellite motion involves estimating the range distance of the object from the observer which is calculated from the assumed emission height. This process is described in detail in an accompanying paper (Wilkins et al. 2017).

\subsection{Requirement Summary}

The ICON FUV instrument is designed to satisfy the following two principal requirements:

(1) ICON shall determine the altitude profile of ionospheric $\mathrm{O}^{+}$plasma density at nighttime through spatial imaging of the oxygen 135.6-nm FUV emissions. The accuracy of this requirement was specified quantitatively by the maximum allowed error in the parameters of an analytic function, which represents $\mathrm{O}^{+}$density as a function of altitude and which is used to model the optical measurements. The error in the altitude of the peak (hmF2) of this function had to be less than $\pm 20 \mathrm{~km}$ and the accuracy of the density at the peak $(\mathrm{NmF} 2)$ had to be better than $10 \%$ when the limb emission was about 20 Rayleighs.

(2) ICON shall be designed to determine the daytime column density ratio of thermospheric $\mathrm{O}$ and $\mathrm{N}_{2}$ using measurements of the altitude profiles of the daytime $135.6 \mathrm{~nm} \mathrm{OI}$ and LBH $\mathrm{N}_{2}$ band FUV emissions. The accuracy of this science requirement was specified with an $8.7 \%$ maximum error in the analytically modeled $\mathrm{O} / \mathrm{N}_{2}$ ratio.

ICON FUV measures limb view optical intensities. These measurements have to be converted into altitude profiles of Volume Emission Rates (VER) of the emitting constituents and then the volume emission rates have to be inverted to density profiles of nighttime $\mathrm{O}^{+}$density and daytime $\mathrm{O}$ and $\mathrm{N}_{2}$ ratio. The preferred method of these inversions relies on forward modeling the volume emission rates profiles and the resultant limb view intensities from assumed analytic functions for the altitude profiles of the densities. Using this technique the limb view intensities were modeled through instruments of varying responsivities to find the instrument responsivity that was required to satisfy the above stated science accuracy requirements. The required horizontal spatial resolution of the measurements was $500 \mathrm{~km}$ and an integration time of 60 seconds was assumed in these calculations. It was found that to obtain the required accuracy of the height (hmF2) and density (NmF2) of the modeled nightside ionospheric F-region peak we needed an SW channel responsivity of at least 13 photo-electron counts $\mathrm{s}^{-1}$ per kilo Rayleigh per second per per $4 \mathrm{~km}$ vertical resolution element. Following the same procedure the daytime $\mathrm{O} / \mathrm{N}_{2}$ ratio accuracy required an LW channel responsivity of 8.3 photo-electron counts $\mathrm{s}^{-1}$ per kilo Rayleigh per second in the LW band per $4 \mathrm{~km}$ vertical resolution element (see Stephan et al., this issue; Kamalabadi et al., this issue) (see Stephan et al. 2017; Kamalabadi et al. 2017). The requirement for the vertical resolution on the limb is intrinsically limited by the atmospheric/ionospheric scale height $(>16 \mathrm{~km})$ at altitudes of 130 $\mathrm{km}$ and above and the $4 \mathrm{~km}$ vertical resolution element (VRE) provided adequate vertical resolution.

The ICON FUV instrument spectral resolution needs to be adequate to resolve the $135.6 \mathrm{~nm}$ spectral line and measure its intensity. In addition it needs to measure the intensity of the LBH emissions of $\mathrm{N}_{2}$ somewhere in the FUV spectral region (Fig. 2). It is clear from Fig. 2 that a moderate wavelength resolution instrument cannot separate the LBH at $135.4 \mathrm{~nm}$ from the $135.6 \mathrm{~nm}$ OI line and therefore a combined measurement of the two will have to be made and the $\mathrm{O} / \mathrm{N}_{2}$ retrieval will have to account for this and other $\mathrm{LBH}$ 
lines within the spectrometer's triangular transmission profile of the SW channel (Fig. 2). Another important spectral requirement is to eliminate the 130.4 OI spectral feature. The limb 130.4 emission is only a few times brighter than 135.6 and a suppression of the 130.4 below $2 \%$ will be adequate. Nevertheless the separation of the 130.4 from $135.6 \mathrm{~nm}$ cannot be achieved with current state of the art multi-layer reflective UV filters and the spectral resolution of a grating instrument is required.

ICON FUV also provides top view images of the ionosphere by the Time Delay Integration (TDI) technique and for these top view images a spatial resolution consistent with a $16 \times 16 \mathrm{~km}$ horizontal sampling was chosen.

The FOV was chosen to be $24^{\circ}$ in the vertical direction, with the central ray pointing $20^{\circ}$ below local horizontal. The following scientific needs drove that selection: (1) Measure emissions in the higher altitude $\mathrm{F}$ region at night up to $500 \mathrm{~km}$ altitude, (2) View the altitude region of FUV extinction by $\mathrm{O}_{2}$ near the middle of the FOV, (3) image a large portion of the sub-limb region. The top edge of the $24^{\circ} \mathrm{FOV}$ was selected to be at 8 degrees below the local horizontal, corresponding to $507.5 \mathrm{~km}$ tangent height altitude from the $\mathrm{S} / \mathrm{C}$ orbiting at $575 \mathrm{~km}$ altitude. The optic axis is aimed at a limb tangent altitude of $156 \mathrm{~km}$. Assuming the sub-limb emissions originate from an ionospheric altitude of $\sim 300 \mathrm{~km}$ (see Fig. 5) this region will occupy the FUV FOV at angles from $32^{\circ}$ to $16^{\circ}$ below the local horizontal, which is equivalent to a viewing region spanning $16^{\circ}$ great circle distance, or $1414 \mathrm{~km}$. There was no explicit requirement regarding the horizontal extent of the FOV other than to make it as large as possible, while simultaneously providing good spatial resolution. We chose it to be $18^{\circ}$.

Imaging of Equatorial ionospheric structures otherwise known as "Spread F" would be optimal if the instrument FOV could be steered to look in the direction of the magnetic meridian. This way the instrument could view the "bubbles" parallel to the magnetic field line. Near the magnetic equator the field is close to horizontal and a northward directed imager has a good chance of imaging the ionospheric bubbles optimally parallel to the magnetic field (Fig. 4). Depending on the location of the spacecraft, the inclination on the orbit and the direction of the magnetic field the field of view may not be wide enough to accommodate viewing angles parallel to the magnetic field. As the multi-instrument nature of the ICON observatory does not permit re-pointing the entire S/C, ICON FUV has a rotating turret with a steering mirror mounted in it to satisfy this requirement over a $\pm 30^{\circ}$ range with $5^{\circ}$ steps. This allows the local magnetic meridian direction to be contained within the FOV of the FUV instrument for most equatorial crossings.

The instrument has to have a large dynamic range to operate in both conditions, when the atmosphere is sunlit observing dayglow and when the atmosphere is in the dark recording the ionospheric nightglow. The limb dayglow of the OI $135.6 \mathrm{~nm}$ line can be 10,000 Rayleighs on the sunlit side whereas only 30 Rayleighs on the dark side. This requires a dynamic range of at least 300 . This requirement is met by using a fast scanning CCD camera of limited dynamic range and digitally co-adding the signal from 100 frames. Since the dynamic range in a single frame is much larger than 3 the overall requirement is easily met. However it might be still desirable to adjust the high voltage from night to day to maximize the instrument operating life time. In the night side conditions the instrument can be run with high gain in a photon counting mode while on the dayside the high voltage on the image intensifier can be lowered to preserve the MCP without any loss in SNR because of the increased statistical noise of the dayside high signal level masks other noise sources.

The instrument requirements are summarized as the Level 3 requirements in Table 1. There is also a requirement for on orbit star calibrations to produce photometric accuracy $15 \%$ or better. 
Table 1 ICON FUV level 3 requirements

\begin{tabular}{lll}
\hline & SW & LW \\
\hline Wavelength & $135.6 \mathrm{~nm}$ & LBH $(150-160 \mathrm{~nm})$ \\
Day & $\mathrm{X}$ & $\mathrm{X}$ \\
Night & $\mathrm{X}$ & Not required \\
Tangent altitude range & $130-450 \mathrm{~km}$ & $130-450 \mathrm{~km}$ \\
Horizontal imaging resolution & $20 \mathrm{~km}$ & $<500 \mathrm{~km}$ \\
Steerable FOV & $\pm 30^{\circ}$ & N/A \\
In-track sampling (12 s exposures) & $100 \mathrm{~km}$ & $100 \mathrm{~km}$ \\
Vertical resolution element (VRE) & $4 \mathrm{~km}$ & $4 \mathrm{~km}$ \\
@ 155 km limb tangent & $0.093^{\circ}$ & $0.093^{\circ}$ \\
Accuracy and precision errors & Modeled hmF2 $=20 \mathrm{~km}$ & Modeled O/N 2 ratio $=8.7 \%$ \\
& NmF2 $=20 \%$ & 8.3 \\
Estimated Responsivity Reqmt. & 13 & \\
(counts s $-1 / \mathrm{kR} /$ VRE) & & \\
\hline
\end{tabular}

\section{The ICON Spectrographic Imager}

\subsection{General Considerations-Imager Responsivity}

FUV imaging is an effective tool for the measurement of the spatial and temporal properties of the upper atmosphere. The definition of the measurement requirements is crucial in selecting a suitable imaging instrument. In this section, we will show that once the science requirements of spatial and temporal resolutions are satisfied by an imaging instrument then the sensitivity or responsivity depends only on the area of the input aperture, A and the overall conversion efficiency, $\varepsilon$ of photons into measurable quanta in the detector. Since these two parameters also define the instruments sensitivity to point sources we will show that calibration of the instrument with point sources is adequate as long as the angular acceptance geometry is also measured separately.

In Fig. 6 a generalized imaging scenario is illustrated. The atmospheric region to be imaged is $R$ distance from the imager on the left of Fig. 6. We identify a sampling area $d a$ that is consistent with the scientific spatial resolution requirements. The instrument gathers all the light from $d a$ entering the instrument aperture $A$ and focuses the light into the area of a science pixel on the detector. On Fig. 6 the imager optics and detector configuration are simply represented by a block with an overall throughput efficiency, $\varepsilon$. This is the efficiency converting photons into measurable information quanta in the detector combining optical transmission and quantum efficiency. There is also a requirement for temporal resolution, which requires the selection of an appropriate collection time and sampling interval, $d t$. The requirement for both spatial $d a$ and temporal requirements $d t$ are defined by the science but it can also depend on the linear and angular motions of the observing platform.

The photon collection in a science pixel during a sampling interval $d t$ is given as:

$$
P_{0}=\frac{10^{6}}{4 \pi} I A \frac{d a}{R^{2}} d t
$$


Fig. 6 A general Imager in which the light from source area, $d a$ is collected into a science pixel

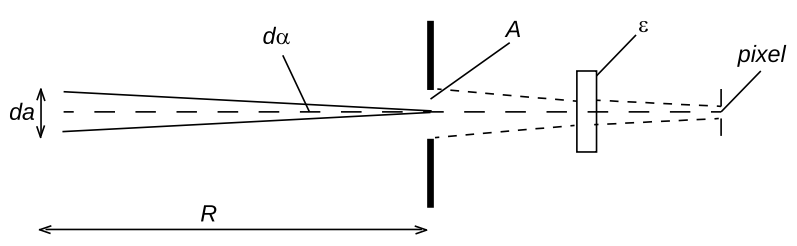

where $I$ is source intensity in Rayleighs, $A$ the input aperture of the imager, $d a$ the area to be resolved, $R$ is the range distance to the target and $d t$ is the sampling time. Since the solid angle $d \alpha=d a / R^{2}$ we can write:

$$
P_{0}=\frac{10^{6}}{4 \pi} d \alpha \cdot d t \cdot A \cdot I
$$

The instrument converts the $\mathrm{P}_{0}$ photons to $\mathrm{P}_{1}$ information quanta with a conversion efficiency of $\varepsilon$ :

$$
P_{1}=\frac{10^{6}}{4 \pi} d \alpha \cdot d t \cdot I \cdot A \cdot \varepsilon
$$

The constant term in (3) $\left(10^{6} / 4 \pi\right)$ is often approximated as 80,000 . The product of terms $I$, $d \alpha$ and $d t$ are independent of the imager instrument choice and they are simply a statement of the scientific requirements of the spatial, temporal and intensity resolution. The choice of the imager defines only the product of the last two terms, $A$ and $\varepsilon$. which is the figure of merit when comparing imager types. It is also sufficient to calibrate an instrument in terms of these parameters provided the angular and temporal resolution of the imager satisfies the science requirements.

The throughput efficiency $\varepsilon$ can be regarded as the product of the optical transmission $T$ and the the quantum efficiency, $q_{e}$-namely the rate of photoelectron production per input photon. The input photon beams have Poissonian intensity distribution and this treatment would assume that the output signal also exhibits Poissonian fluctuations. In an efficient system this should be approximately true however strictly speaking we should take into account other noise sources and calculate the true equivalent quantum efficiency $q_{e q}$ which is the ratio of the square of the signal to noise at the output signal, ${\overline{\nabla P_{1}}}^{2}$ divided by the photon noise squared at the input, ${\overline{\nabla P_{0}}}^{2}=P_{0}$. This is discussed in more detail in Sect. 5.8 entitled "Detector performance".

The responsivity of an instrument is usually given in counts Rayleighs ${ }^{-1} \mathrm{~s}^{-1}$ which is from (3):

$$
\frac{P_{1}}{I}=\frac{10^{6}}{4 \pi} d \alpha \cdot A \cdot T \cdot q_{e}
$$

The responsivity value needed to achieve the scientific accuracy and precision requirements was discussed in Sect. 2.3 "Requirement Summary".

The responsivity of an instrument can be measured directly through calibration when a known intensity (equivalent Rayleighs) extended source is placed in front of the instrument and the signal strength is measured. Alternately the responsivity in (4) can be derived from a mechanical measurement of the input aperture area, $A$, the angular resolution of pixels $d \alpha$ from geometric calibration and the instrument throughput efficiency, $\varepsilon=T q_{e}$ from the instrument response to a collimated light source. Using these quantities and measuring the input aperture, $A$ and the solid angle of acceptance of a resolution element, d $\alpha$. Hence the responsivity can be calculated from (4). This latter technique was employed in estimating the extended source responsivity of the ICON FUV instrument. 
ICON FUV will also rely on inflight calibrations using known intensity UV stars. The star calibrations are another example of point source calibration. It is necessary to have very good measurements of the relative spectral responsivity of the instrument because the star calibrations depend on the star's known brightness which has to be convolved with the instrument spectral responsivity.

\subsection{The FUV Spectrographic Imager}

Two concepts are commonly used in FUV aeronomy: (1) Imaging Spectrometers (IS) and (2) Spectrographic Imagers (SI) (Mende 2016). In both instrument types the dispersing element, the grating operates in combination with a slit to select the wavelength pass-band. The slit can be regarded as a mask, selecting only certain angles to pass. However, the image is formed at the slit in the IS while the image is formed at the grating in the SI. In the IS spatial imaging at the output is a convolution of the image and the instrumental wavelength profile. This is contrary to the SI where the spectral dispersion and the imaging properties are in "quadrature", i.e. they are separate and independent of each other.

In Fig. 7, we present a schematic illustration of a dual wavelength SI concept using refractive optical elements. The diagram provides two illustrations of the ray-paths through the same instrument. The top is the ray path shown for spectral selection. Light enters at the slit and is collimated by the collimator optics that are placed one focal distance behind the entrance slit. This collimating optics has two basic functions, first it produces parallel light from the slit and second, it focuses the viewed object on the grating. The parallel light is dispersed by the grating according to wavelength. By having two separate exit slits it is possible to have two separate wavelength channels in a single instrument. In Fig. 7, the wavelength

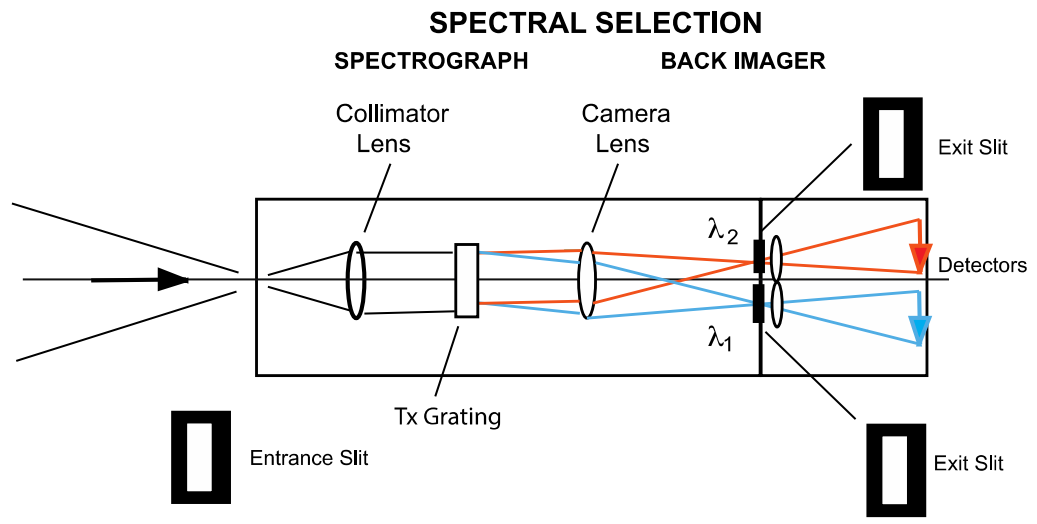

IMAGING

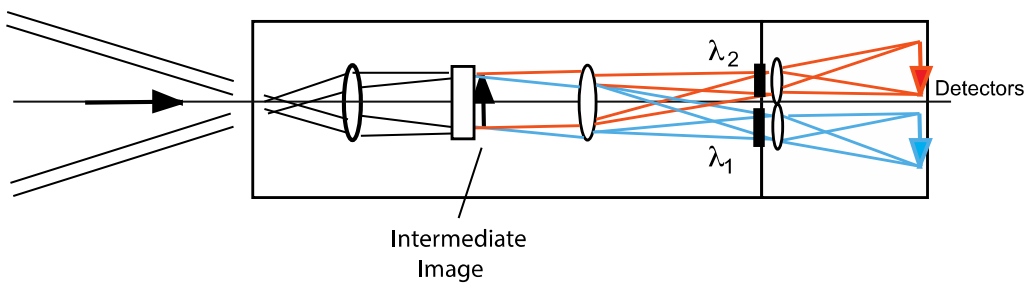

Fig. 7 Schematic of the optical principle of a spectrographic imager designed to accept two wavelength channels using lenses 
Fig. 8 A view of the ICON FUV instrument on the bench

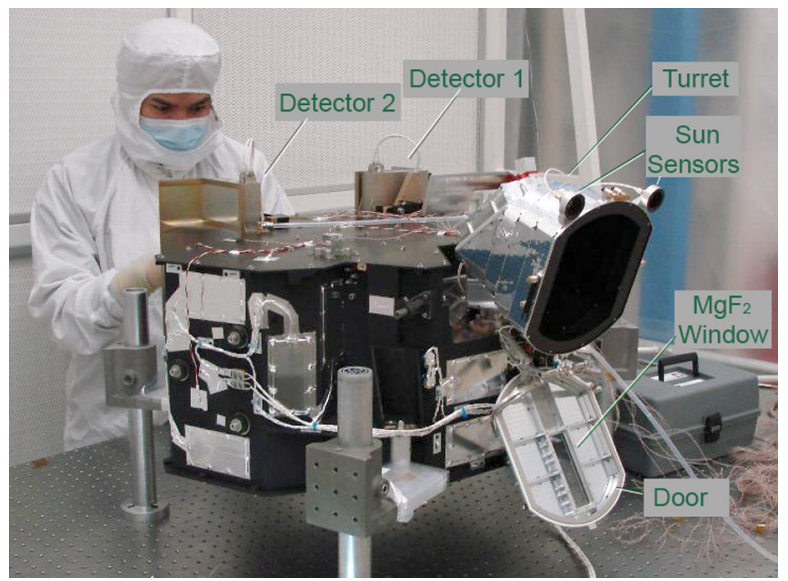

$\lambda_{2}$ illustrated in red dispersed upward and the other wavelength $\lambda_{1}$ illustrated in blue is dispersed downward. After passing the grating, the camera lens focuses the parallel blue light of wavelength $\lambda_{1}$ into the lower exit slit, while the red light of wavelength $\lambda_{2}$ is focused into the upper slit. The blue and red light reach separate detectors in our scheme. In terms of the upper diagram, the instrument can be regarded as a conventional monochromator with two exit slits and without imaging. The lower illustration of Fig. 7 represents the same optical train, but shows how two dimensional imaging takes place. From each distant object point in the scene, parallel light enters the entrance slit. The collimator lens, which is placed one focal distance in front of the grating, focuses the parallel light on the grating, thus forming an intermediate image at the grating. The "camera lens", following the grating in combination with the small lens placed behind each exit slit re-images the intermediate image on the detector. This instrument therefore produces two-dimensional spectrally filtered images of the same scene on two detectors simultaneously. These instruments were introduced in the ultraviolet for space use where narrowband filtering with multi-layer filters would have been otherwise problematic (Mende et al. 2000c).

The first example of a Spectrographic Imager was flown on the NASA IMAGE mission. In this instrument the grating spectrometer was a Wadsworth configuration instrument with a concave grating and with a hole in the grating center where the entrance slit was placed. This allowed the use of an axial collimator for improved spectral performance. However, this hole appeared as an obscured region at the center of the final image. For ICON it was considered highly desirable to remove the obscuration and a Czerny-Turner (CZT) (Czerny and Turner 1930) spectrograph configuration was selected instead. With the ICON resolving requirements, this optical arrangement permitted the use of off-axis mirrors and eliminated the requirement for the central obscuration of the IMAGE Wadsworth type instrument. A disadvantage of the Czerny Turner-based instrument is that it requires an additional mirror with the associated reflective losses and mass and cost penalties. Considerable progress has been made in producing highly-efficient UV mirrors since the IMAGE program and the additional reflection loss became a less crucial issue (Quijada et al. 2012).

A photograph of the ICON Spectrographic Imager is shown on Fig. 8. A top view of the optical model is illustrated in Fig. 9. The viewed object is schematically illustrated by an arrow on the top. Light enters the instrument at the top of the illustration and is reflected by the steering mirror. The periscopic steering mirror points the direction of the optic axis by rotating around a vertical (perpendicular to the page) axis. Actually there are two small $45^{\circ}$ 


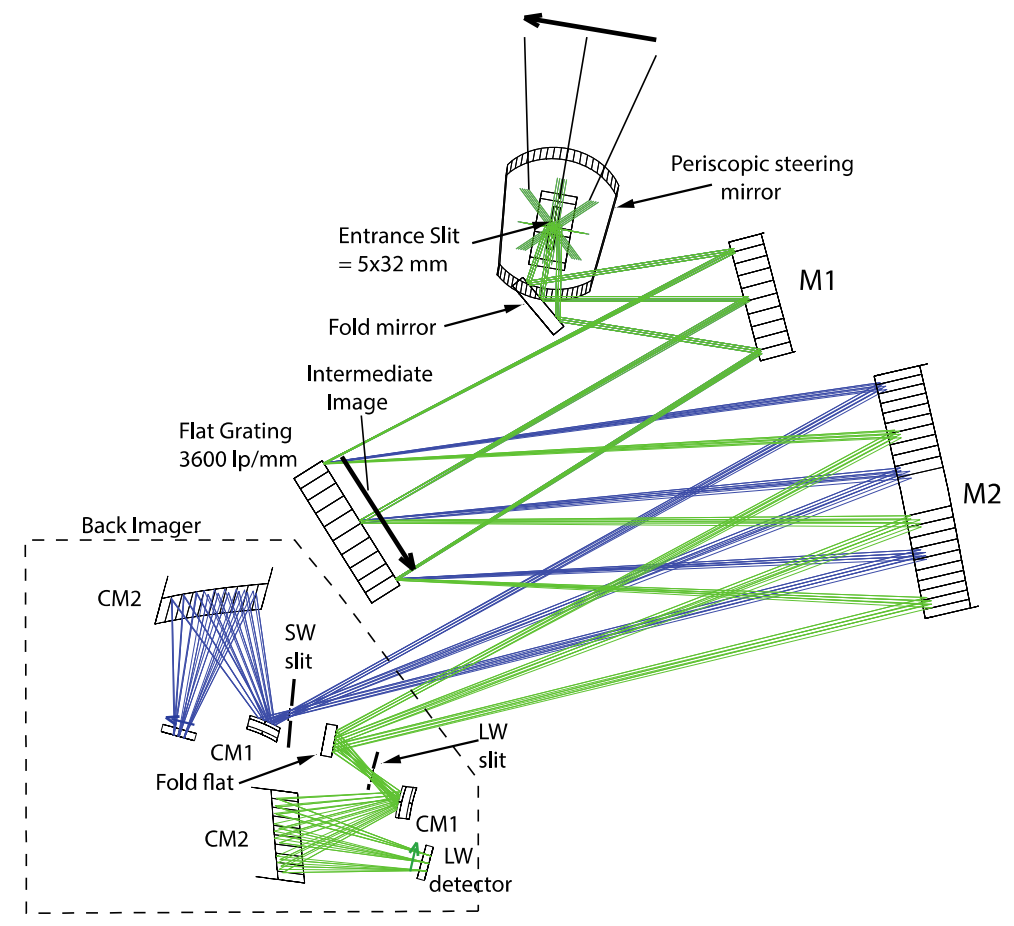

Fig. 9 Top view schematic of the ICON FUV imager

mirrors behind the entrance slit, one of them is to provide the second periscope mirror and the other to turn the image to ensure that that the horizon is perpendicular to the slit when the turret is in its normal (turret angle $=0$ ) position. These two mirrors are not shown on the schematics. The spectrograph slits, grating and detectors are fixed in the instrument frame but if the scan mirror is not in its normal position the projection of the slit on the outside view will appear to be rotated. Thus effectively the spectral slit will be superposed on the outside atmosphere at an angle with respect to the vertical depending on the position of the steering mirror.

All optical elements are reflective with the exception of the detector windows, which are $\mathrm{MgF}_{2}$. The spectrograph mirrors M1 and M2 are both spherical. M1 acts as a spectroscopic collimator as well as a camera mirror to create the intermediate image of the scene at the grating acting the same as the collimating lens in Fig. 7. M2 is the spectrograph's "camera mirror," focusing the image of the input slit at the output slits. Depending on the wavelength, the grating dispersion and the location of the output slits, light of the appropriate wavelength band is selected. One can regard M2 as a collimator for the imaging operation, creating nearparallel light for each image point on the grating to be re-focused on the detectors by the back imager aspheric mirrors CM1 and CM2. There is a separate set of back imager optics for each of the two wavelength channels (SW and LW) including the exit slits shown in black. In order to allow more room and accommodate the configuration, the LW channel has a turning flat mirror, which allows placing the LW channel out of the way of the other channel.

A dimensioned drawing of the ICON FUV top view and two side views are presented as Fig. 10. The technical resource requirements are documented in Table 2. 


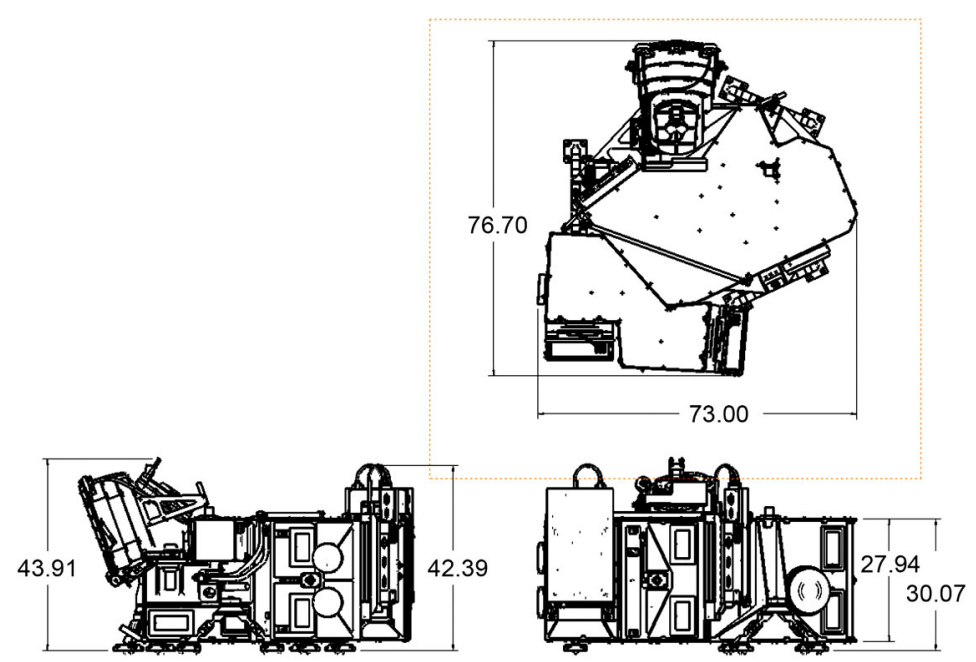

Fig. 10 Dimensions and layout of the ICON FUV instrument. All dimensions are in $\mathrm{cm}$

Table 2 ICON FUV resource summary

\begin{tabular}{lll}
\hline Property & Quantity & Units \\
\hline Mass & 33.29 & $\mathrm{~kg}$ \\
Orbital average power & 9.58 & Watts \\
Stepper motor & 4.9 & Watts \\
Operating T. range & -13 to 41 & ${ }^{\circ} \mathrm{C}$ \\
Survival T. range & -25 to +43 & ${ }^{\circ} \mathrm{C}$ \\
\hline
\end{tabular}

\subsection{ICON FUV Detectors, Electronics and Data Handling}

The ICON FUV detectors consist of image tubes that are fiber-optically coupled to CCDs. The image tubes have $\mathrm{MgF}_{2}$ windows and FUV photocathodes evaporated directly on to the microchannel plate (MCP). A stack of two MCPs are used, which arrangement provides sufficient charge multiplication gain to overcome readout or dark current noise downstream in the CCD. The CCDs operate in a fast scan mode at 8.33 frames per second (fps). 100 video frames are co-added digitally in memory in the ICON Instrument Control Processor (ICP) to produce images of 12-second integration. The data from the 12-second exposures are downlinked to the ground. Because the satellite moves substantially during 12 seconds, two types of motion compensation schemes are used. In type one, six horizontally co-added vertical altitude profiles are generated for the measurements of the altitude distribution of the emission intensity (Fig. 11). In this figure the image of the limb is shown to be projected on the detector between tangent heights of about 150 and $507 \mathrm{~km}$. The data is co-added horizontally into six strips as shown in blue. These strips represent the primary data source for taking vertical profiles of the thermosphere/ionosphere. The image can be regarded as having "rescells" of $4 \times 4 \mathrm{~km}$ at the tangent height of $155 \mathrm{~km}$ representing an angular region of $0.093 \times 0.093$ degrees. Approximately 32 of these rescells are co-added in one stripe and each stripe represents 3 degrees in horizontal width. The rescells nominally would translate to a $4 \times 4$ binned CCD pixel on a $256 \times 256$ matrix of the detector however the imager optics has substantial non-linearity that has to be accounted for. A single strip is illustrated 


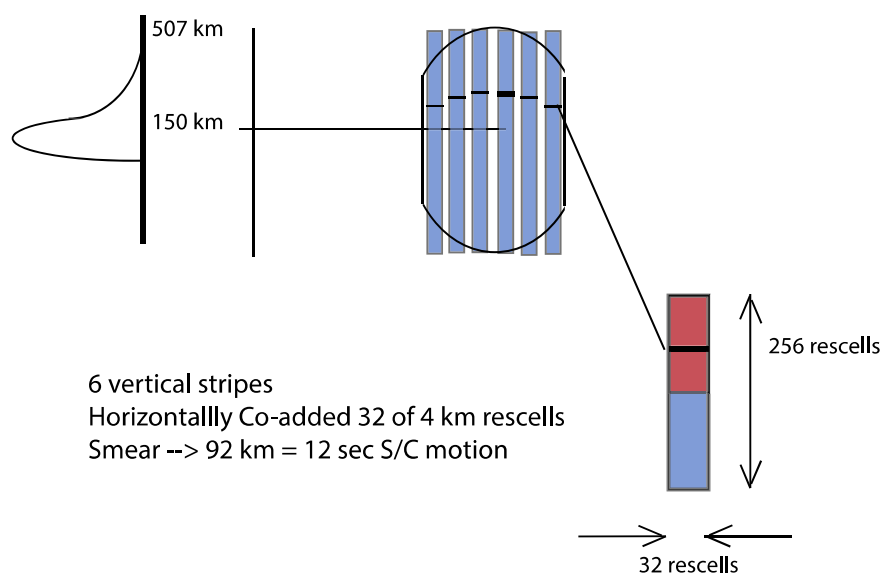

Fig. 11 Illustration of limb altitude profiles. There are 6 vertical strips. In each strip the pixels are co-added horizontally. During daytime data is taken for both channels while during nighttime only for the $135.6 \mathrm{~nm}$ channel. Below $150 \mathrm{~km}$ altitude there is substantial $\mathrm{O}_{2}$ absorption in the FUV and it is not possible to get limb views of the atmosphere. The linear dimension of a "resolution element" or "rescell" is $0.093^{\circ}$ or $1 / 256$ of the $24^{\circ}$ vertical FOV

on the right and the limb view region is shown in red. Below $130 \mathrm{~km}$ tangent height the instrument is viewing a region where all FUV emissions in the background are absorbed by $\mathrm{O}_{2}$ and this "sublimb" or "disc" region is illustrated in blue. The six profiles are generated by summing pixels horizontally in the direction of satellite motion without smearing in the vertical direction. The co-adding process is relatively straight forward when the turret is in its baseline $\left(0^{\circ}\right)$ position and the horizontal direction in the image of the limb is parallel with the pixel $x$-coordinate in the image. When the turret is at another angle, the co-adding of pixels has to take place along a slant path in the imager frame of reference. This is accomplished by transposing the images and modifying the pixel addresses during the transposition, thereby removing the geometric distortion and applying an $x$ and $y$ pixel shift to facilitate co-adding along contours that represent horizontal paths. The pixel addresses for the transposition use an address matrix stored in a Look-Up-Table (LUT).

The second type of motion compensation scheme produces a data stream containing Time Delay Integrated (TDI) images reproducing a mapped view of a two dimensional horizontal intensity distribution (Wilkins et al. 2017). In this mode, the individual frames are digitally co-added in the ICP memory after they have been projected on an imaginary horizontal surface, which is moving uniformly in the satellite frame of reference (Fig. 12). During the co-adding process, a constant offset is applied to the address of each pixel in each frame to shift the image. This offset is computed from the orbital speed of the satellite so that it compensates for the satellite motion.

During nighttime the $135.6 \mathrm{~nm}$ emission maps will be treated as two dimensional images viewed from above. The $135.6 \mathrm{~nm}$ emission is produced by recombination of $\mathrm{O}^{+}$and its intensity is expected to peak at the bottom of the F region at an altitude of approximately $300 \mathrm{~km}$. We divided the view of the atmosphere into two regions. Imaging at elevation angles corresponding to ray tangent height of $300 \mathrm{~km}$ or higher we map the emissions to the appropriate limb tangent because we expect the greatest intensity to be seen there and these images are called as "limb view" images. At elevation angles lower than the limb tangent of $300 \mathrm{~km}$, we map the layer to a constant altitude of $300 \mathrm{~km}$ below the satellite as "sub-limb" views. We recognize that there is a confusion in the region from tangent heights of 150 to 


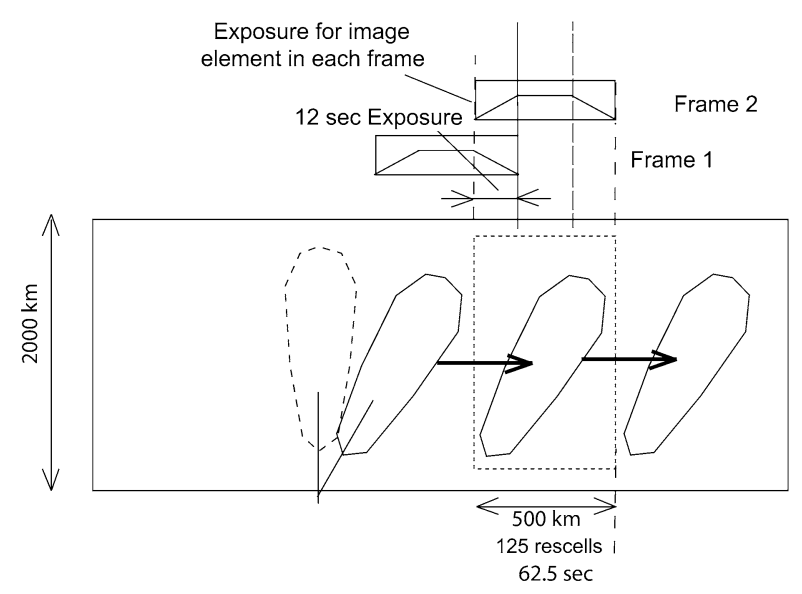

Fig. 12 A schematic of TDI co-adding of limb or sub-limb images as the satellite is moving from left to right. The turret angle is illustrated to be $30^{\circ}$ forward. In consecutive exposures some pixels near the edges of the FOV are only partially exposed whereas pixels in the middle receive full exposures. The exposure time of each image element, depending on their position in the field, is shown with the small trapezoidal illustration. Adjacent frames fill in the exposure time of regions near the edge of the FOV. The complete sequence of exposures with appropriate superposititon of the images can be used to reproduce all pixels at their full exposure

$300 \mathrm{~km}$ between structures originating nearer than the tangent point and structures further away from the tangent point. Both of the limb view and sub-limb models define an altitude and a range distance from the satellite and therefore can be mapped as a function of their horizontal position.

Consecutive exposures using the TDI technique can be summed up to a single continuous map of the limb-view and sub-limb images along the orbit. We take 12 second exposures, which is shorter than the travel time of image elements across the FOV. Imaged elements in the middle of the FOV get a full 12 second exposure, however elements at the forward and backward edges of the FOV get only limited exposures. This is illustrated on Fig. 12 where the small trapezoids represent the exposure time of each image elements. The purpose of the illustration is to show that elements at the edge of the FOV will get the same exposure time when consecutive exposures are summed up in the post flight data analysis.

This TDI technique provides high-resolution images in spite of the substantial motion of the satellite platform during the 12-second exposure. It should be noted that these techniques require sophisticated real-time image manipulations onboard the satellite. This is accomplished in ICP resident Field Programmable Gate Arrays (FPGAs) using firmware because of the high processing speeds needed. This technique is an evolution of the TDI motion compensation that was used in the FUV instrument on the NASA IMAGE satellite (Mende et al. 2000a), with a much more detailed set of performance requirements (Wilkins et al. 2017, this issue).

\subsection{ICON FUV System and Electronics}

The ICON FUV system is illustrated in Fig. 13. In addition to the opto-mechanical instrument structure, the ICON FUV flight system also has some dedicated electronics components resident in the Instrument Control Processor (ICP). The instrument operation is controlled and the CCD image data is processed on board by the ICP electronics. There is a 


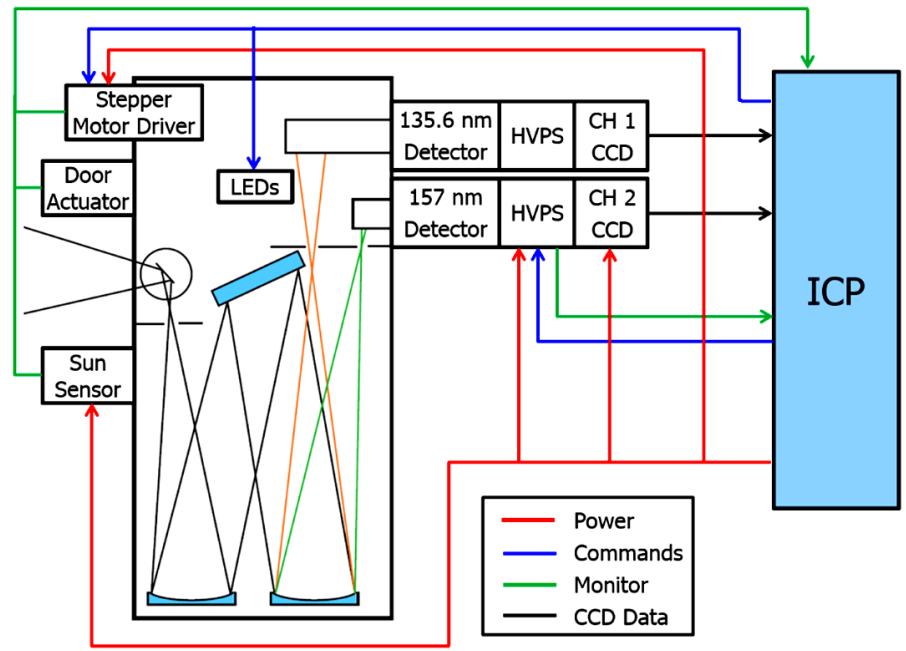

Fig. 13 The ICON FUV system diagram. On board signal processing takes place in the Instrument Control Processor (ICP)

wiring harness connecting the ICP to the rest of the instrument. The harness contains power and data connections and provides control commands to each of the ICON FUV sub systems. The camera intensifier high voltage power supply voltages are commanded by the ICP. The sun sensor sends signals to the ICP to set the high voltage to a safe level when the sun comes close to the FOV. The high voltage control is all direct hardware with quick response. There is a contamination cover door in front of the baffle which opens in orbit one time only. This cover protects the scanning mirror from deposits of hydrocarbon and particulate contamination. The stepper motor driver controls the turret position. There are UV LEDs in the back imager cavities, which can be used to stimulate the detectors by flooding them with a quasi-uniform illumination. These LEDs operate in the near-UV wavelength region and can work in air. This stimulus system verifies the integrity of the entire detector chain thus providing great confidence about the condition of the detector system and associated electronics during all ground based testing,

\section{ICON FUV Development and Testing}

The ICON FUV instrument is based on the Spectrographic Imager which was flown on the NASA IMAGE satellite. The build of that instrument started in 1996. The design concept and the original ray tracing were developed at UC Berkeley. The detailed optical design and the design of the opto-mechanical parts were performed by the Centre Spatiale de Liege (CSL) in Belgium. CSL took a then novel approach and built the flight instrument relying entirely on computer models without first building any kind of an engineering model. Back in those days building of an engineering model and testing it prior to building the flight model was the conventional customary practice. CSL did extensive modeling of the optical and mechanical systems before cutting any metal. The IMAGE spectrographic imager was a great success and operated on orbit exactly as predicted. Although the roles of CSL and Berkeley were different in the ICON build, the same systems-modeling followed by flightbuild approach was adopted for ICON. 
Table 3 Comparison between the flight UV-grating versus a visible grating that simulates the same operating geometry

\begin{tabular}{llll}
\hline & $\begin{array}{l}\text { Ruling density } \\
\text { lines/mm }\end{array}$ & $\begin{array}{l}\text { Channel 1 } \\
\text { Center of pass-band, nm }\end{array}$ & $\begin{array}{l}\text { Channel 2 } \\
\text { Center of pass-band, nm }\end{array}$ \\
\hline Flight grating & 3600 & 135.6 & 157.00 \\
Visible grating & 900 & 542.4 & 628.00 \\
\hline
\end{tabular}

\subsection{Visible Alignment and Testing}

There is a fundamental difficulty in the integration and especially the alignment of vacuum ultraviolet instruments because they only work in vacuum and it is very difficult to tune them up without making some special arrangements, which minimize vacuum alignment and focusing. ICON FUV followed the same path as IMAGE FUV and the mounting of some critical elements were instrumented so that they could be manipulated remotely from outside of the vacuum chamber. In the ICON instrument the mirror M1 in the spectrograph and both CM2 mirrors were mounted on motorized screw mounts so that they could be aligned while they were in the vacuum chamber. It was planned that when the system was fully aligned the vacuum chamber would be brought to atmospheric pressure and the moveable mounts would be clamped and staked in position.

A major risk reduction effort was undertaken in aligning the optical flight system in air, using visible light with manual access to all components. This approach let us test and develop the procedures necessary for the UV focusing activity in vacuum. The grating and the detectors were the only wavelength-dependent optical components. A grating ruled for visible light and visible GSE cameras were substituted for the flight parts in this process. Table 3 shows the operating wavelength which were used to align and tune up the instrument in the visible wavelength.

To facilitate the visible tests we used an image projector using a wide field angle fast photographic camera lens, which could be regarded as a super wide field collimator producing parallel light from a test pattern over the entire angular ICON FUV FOV. We used a large format visible CCD camera with a thick window to ensure that the optical path to the camera CCD was the same as it was to the photo-sensitive element of the FUV detectors. Figure 14 is an image taken at the exit aperture of the LW channel. The image shows the optical field as it appeared on the CCD in the red $628 \mathrm{~nm}$ light. It is possible to discern the closely spaced grid of the dot pattern which was a part of the test pattern in the image projector. This was a confirmation that the instrument design was working and that we could achieve focus using the electrical actuators designed for vacuum. One of the benefits of this exercise is that we found that the act of clamping after adjustment could actually displace the optical elements thereby changing the wavelength selection and imaging performance. Knowing this, we were able to develop a clamping procedure, which minimized the displacement. This same procedure was later used after the instrument was focused and optimized in the vacuum chamber with FUV light. It would have been very time-consuming to align the instrument with FUV light without this procedure because we would have had to pump down after each adjustment to perform the post clamping optical testing. After finishing the visible testing program the instrument was shipped to Belgium where the FUV alignment, functional and environmental testing and calibration would take place. The instrument was shipped with the visible grating in place so that it could be re-tested in Belgium. The 3600 lines $/ \mathrm{mm}$ flight grating was tested (Blain et al. 2016) and installed with the flight detectors in Belgium. 
Fig. 14 LW channel image of a test pattern illuminated with $628 \mathrm{~nm}$ light while the non-flight 900 lines/mm grating was installed in ICON FUV
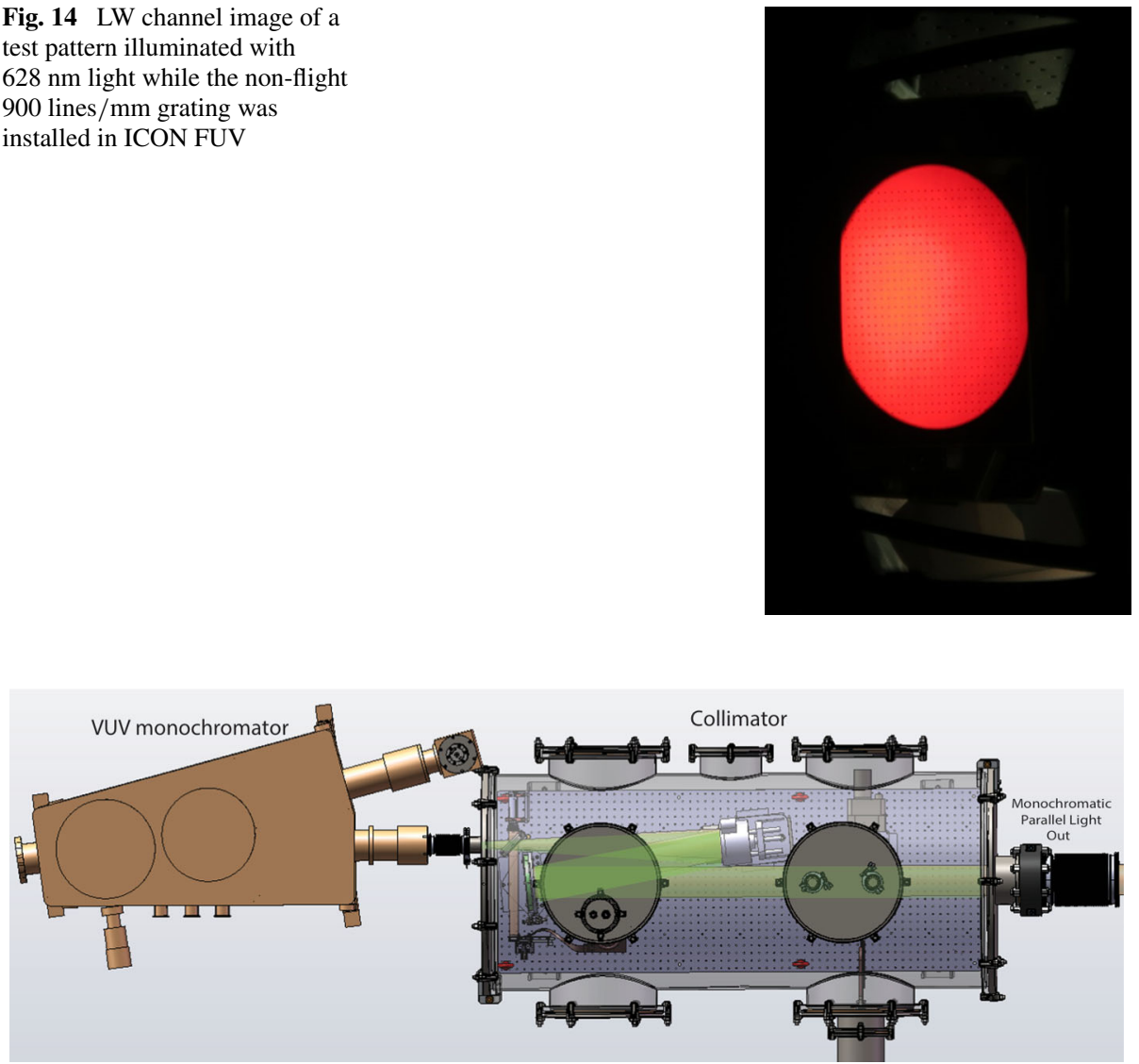

Fig. 15 CSL vacuum UV monochromator and collimator. The monochromator exit slit is in the focal plane of the collimator optics and the collimator produces parallel light

\subsection{FUV Alignment, Testing and Calibration}

CSL constructed a sophisticated FUV testing facility for the ICON FUV alignment testing and calibration (Loicq et al. 2016c)

The VUV alignment and calibration was performed inside the 6.5 meter diameter round vacuum chamber at CSL in Liege. The light source used in the tests was a high-pressure deuterium lamp on a McPherson vacuum monochromator coupled to a two component off axis collimator shown in Fig. 15. The parallel light from the collimator entered the large vacuum chamber, which housed the ICON FUV instrument mounted on its 3 axis articulating cradle (Fig. 16). The instrument was mounted sideways so that the rotation around the cradle horizontal axis allowed the compensation of the beam angular displacement by the turret rotations $\left( \pm 30^{\circ}\right)$. Rotation of the cradle around its vertical axis allowed movement of the image in the up down direction $\pm 12^{\circ}$.

The sequence of tests were as follow, visible alignment, UV alignment and clamping, vibration tests, post vibration focus test. These tests were followed by thermal vacuum testing coupled with calibrations at various temperatures. 
Fig. 16 ICON FUV mounted on its side on the articulating cradle inside the vacuum chamber. Turret and contamination cover door are nearest to the reader

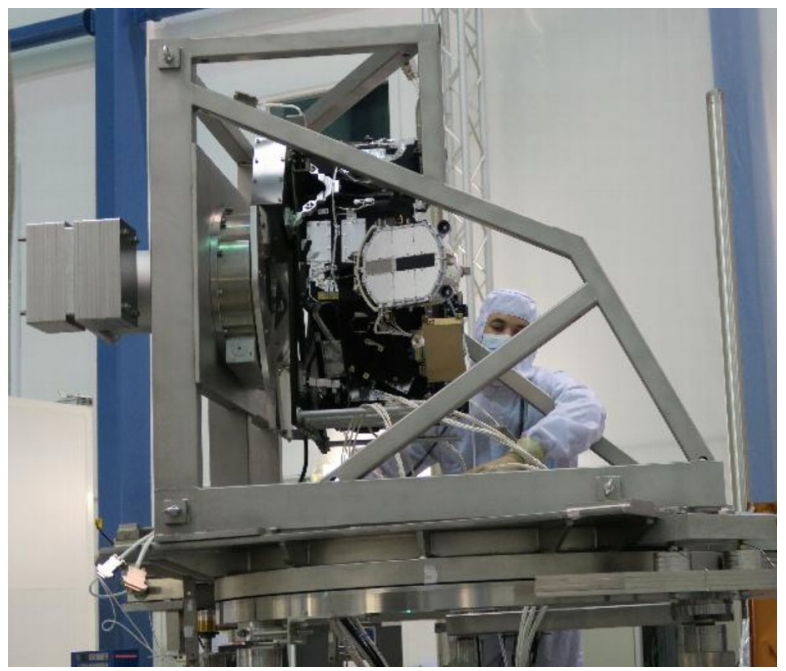

\section{ICON FUV Detailed Design and Performance}

\subsection{Optics}

The general instrument requirements were discussed earlier regarding FOV, pixel size, and wavelength coverage. A detailed discussion of the optics can be found in Loicq et al. (2016a, 2016b). The ray tracing of the instrument is presented in Fig. 9.

The optical design optimization was performed with CODE V and ASAP optical ray tracing software. The instrument can be functionally separated at the exit slits into the CzernyTurner (CZT) and the two back imagers (Fig. 9). It can be shown that the back-imager imaging properties are not much impacted by the CZT spectrograph. Nevertheless the imaging properties of the CZT forming the image on the grating also needs to satisfy the imaging requirements and be compatible with the back imagers. This requirement should not be too difficult to meet because the image on the grating is large and the convergence angles of the rays are therefore small due to the conservation of etendue. Therefore we expect that the intermediate image formation of the CZT is much less demanding here than at the back imagers. The back imager optics has no influence on the spectral selection. Consequently the optimization process can be done independently for the CZT and for the back-imagers.

At the start of the program, the original CZT design used two reasonably similar size spherical mirrors M1 and M2 combined with a convex spherical grating. It was soon recognized that making high line density convex gratings was a novel requirement and that it could be problematic. Therefore a planar grating was substituted in the design. This approach is the simplest implementation of a CZT, however it suffers from astigmatism due to the different focal lengths in the tangential and sagittal planes resulting from the off-axis reflections from the spherical mirrors. It was important to minimize the astigmatism of the CZT as much as possible at the exit slit plane. This effect is well described in Dalton (1966), Bates et al. (1970) and Austin et al. (2009). One simple method was to compensate for this by reducing the distance between the input entrance slit and the collimating mirror so that the grating is under divergent illumination (Bates et al. 1970). In this condition, diffraction in the tangential plane upon reflection from the grating introduces an astigmatism that can 
compensate for the off-axis reflections from the spherical optics. As a result of these considerations the CZT design became quite asymmetrical with a relatively small M1 and a very much larger M2 (Loicq et al. 2016a, 2016b). Unfortunately, the accommodation of the large M2 proved to be mechanically difficult because it necessitated the mounting of all optical elements at a large distance from a conventional optical bench. The end result was that the system was designed so that the components would be mounted in a rigid box structure without an optical bench.

The CZT spectrograph design was first optimized by considering the entrance slit as a set of point sources whose emitted angular beams cover the full FOV. On the exit slit plane the imaged spot sizes through the CZT had to be minimized for both science wavelengths. The free optimization parameters are the M1 \& M2 curvatures, with the goal to keep them constant, position $(x, y, z)$, and tilt. The merit function for the instrument is the energy throughput as a function of wavelength when considering all the whole FOV. The instrument optimization consisted of maximizing this function at the central wavelength and minimizing it for wavelength to be rejected.

The flight and flight spare gratings were replica gratings of a holographic master made by Horiba in France. The gratings were coated at the NASA Goddard Center coating labs with their special high temperature $\mathrm{MgF}_{2}$ coating process (Quijada et al. 2012).

\subsection{Wavelength Selectivity}

The entrance slit size plays into a performance trade between etendue and spectral resolution. The slit size had to be consistent with the grating ruling density and the focal length of CZT mirrors M1 and M2. The ruling density choice was driven by a maximum ruling density consistent with manufacturing capabilities of grating vendors. It was chosen to be 3600 lines $/ \mathrm{mm}$. The grating had to be homogeneous in diffraction efficiency over the full size of the intermediate image corresponding to the full field of view. Even at this ruling density the project was not able to obtain a "blazed" grating, one in which the shape of the rulings are modified to enhance reflectivity at the desired wavelength. Two holographically ruled flight gratings were procured from Horiba for ICON FUV. The IMAGE project Spectrographic Imager gratings were procured some 15 years earlier and they were also holographically ruled at 3000 lines/mm by Zeiss but they were blazed by ion etching and therefore they were about a factor of two or more efficient. It is regrettable that the technology for ion etching was not readily available for ICON FUV in 2013.

The first criterion to meet was the CZT's spectral selection. The spectral selection requirements for each channel were different. Figure 2 describes the spectrum of dayglow in the FUV. The SW channel is mainly dedicated to the oxygen line centered at $135.6 \mathrm{~nm}$ whereas LW is dedicated to several $\mathrm{N}_{2} \mathrm{LBH}$ bands around $157 \mathrm{~nm}$. Lines to be rejected were: $121.6 \mathrm{~nm}$ Hydrogen Lyman alpha, $130.4 \mathrm{~nm}$ atomic oxygen line, 149.3, $\mathrm{nm}$ atomic nitrogen line and the 164.1 atomic oxygen line. The importance of a good focus of the image of the entrance slits at the plane of the exit slit is illustrated in Fig. 17. In Fig. 17a we show what happens when the image of the entrance slit is defocused by moving M2. When M2 is defocused, it is difficult to place a large area exit slit so that it accepts only the region containing $135.6 \mathrm{~nm}$ emission and at the same time rejects all 130.4. In Fig. 17b we illustrate that when the image of the entrance slit is well focused for both 135.6 and 130.4 then it is reasonable to have the two regions well resolved side by side. The result of scanning a perfectly rectangular profile of the image of an entrance slit over another perfectly rectangular profile of an exit slit yields a triangular throughput function. Thus the predicted wavelength response of the SW channel has a triangular shape with a FWHM of 4.8nm shown in Fig. 18. 
Fig. 17 (a) SW fields footprint on the $135.6 \mathrm{~nm}$ exit slit plane as a function of moving M2 and thereby defocusing the instrument. (b) SW footprints at 135.6 compared to the 130.4 footprint which has to be rejected. The gap has to be large enough to keep the two footprints separate and guarantee the 130.4 spectral rejections

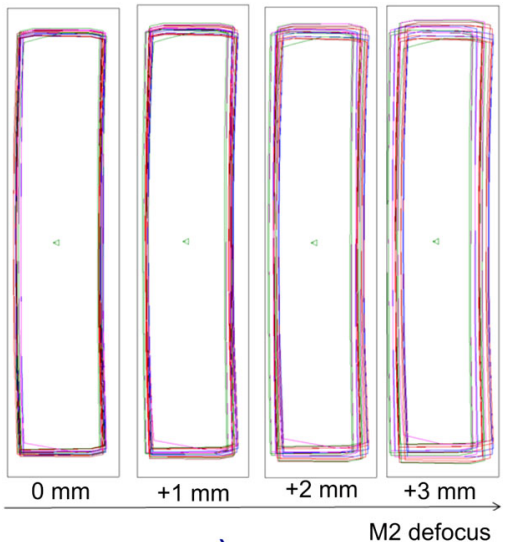

a)

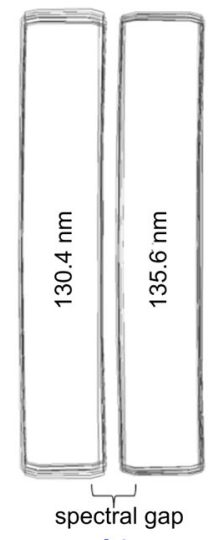

b)
Fig. 18 Spectral profile of the SW channel analyzed at the exit slit plane. It shows that the design is working well in suppressing $130.4 \mathrm{~nm}$ emission

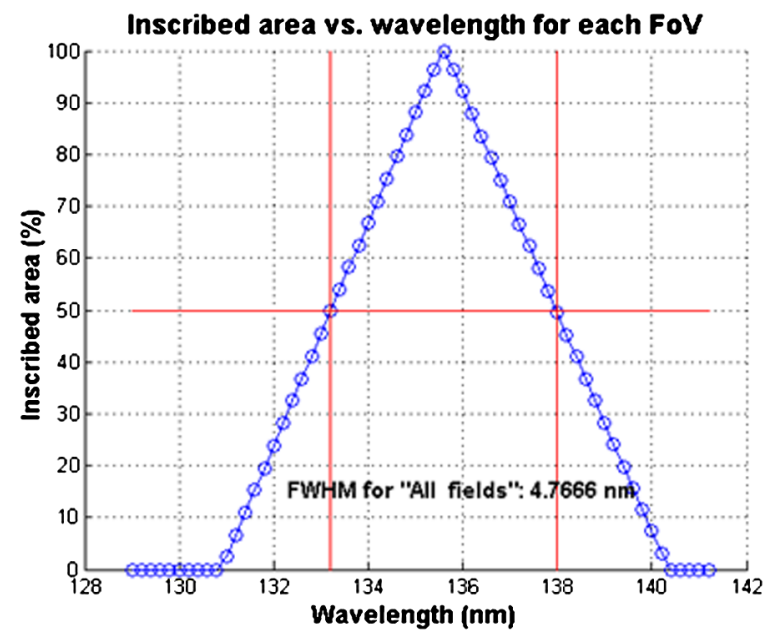

The actual measured wavelength profile is shown in Fig. 19. This measurement was taken during instrument calibration (Loicq et al. 2016b). The detector signal measurements are shown in black. The measurements had to be normalized because the source intensity had a strong variation with wavelength depicted with the dotted line. The resultant normalized transmission profile is shown in blue. A Gaussian profile was fitted to the result and the central wavelength position of the Gaussian is shifted $0.33 \mathrm{~nm}$ towards the high wavelength side from $135.6 \mathrm{~nm}$ and the full width at half maximum (FWHM) is $4.73 \mathrm{~nm}$. The measurements (asterisks) show that the response was too small to measure at wavelengths of $130.4 \mathrm{~nm}$ and shorter.

On the LW channel the main objective was to maximize the LBH light flux coming into the instrument. The spectrometer focus of channel 2, which determines its wavelength resolution, is less critical because the nearby contamination lines 149.3, and 164.1 are much dimmer than 130.4 or Lyman alpha. To pick up more energy from the LBH molecular band system a larger wavelength window was desirable and the exit slit of the LW channel was wider than the SW exit slit. 


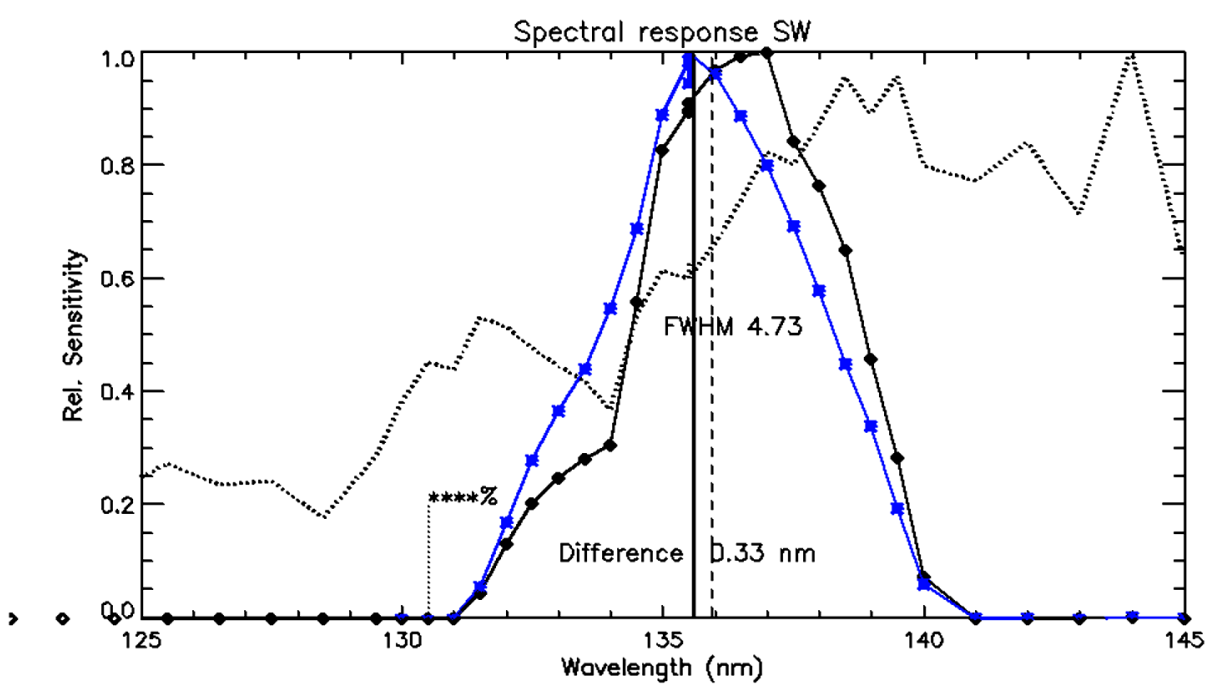

Fig. 19 Measured wavelength response of the SW channel in the flight instrument. Black curve: measured profile normalized to the maximum counts. Dotted line curve: source signal ( $I$ in nA at a photometer measuring the input flux at the entrance aperture of the instrument) normalized to maximum current. Blue curve: measured signal divided by source current $(P / I)$ normalized to maximum. Solid vertical line: $135.6 \mathrm{~nm}$, dashed vertical line: center line of a Gaussian fit to the blue curve and the "Difference" is between $135.6 \mathrm{~nm}$ and center of the Gaussian FWHM is given for the fitted Gaussian (Color figure online)

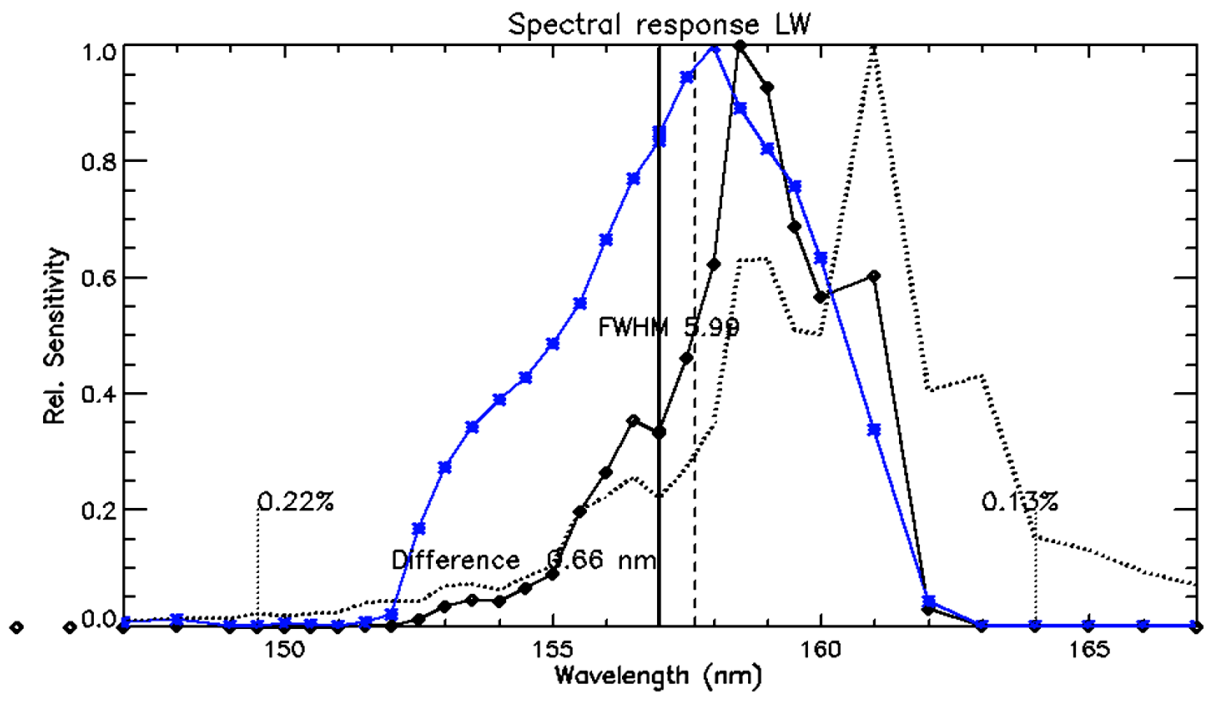

Fig. 20 Measured wavelength response of the LW channel in the as built instrument. Same as Fig. 19 except that short dotted vertical lines mark the unwanted 149.3 and 164.1 features and their percent of that signal with respect to the maximum normalized signal (Color figure online)

The calibrated wavelength response of the LW channel is shown in Fig. 20. The Deuterium light source feeding the optical GSE is quite variable in this spectral range (dotted line). There is a strong emission feature in the spectrum around $170 \mathrm{~nm}$. The measured 
Fig. 21 SW channel spot shapes and sizes with related fields. The position is indicated as fraction of the field (vertical and horizontal) and degrees (DG) underneath. For scale a 50 micron horizontal bar is indicated at bottom right. The r.m.s spot sizes are indicated as RMS in $\mathrm{mm}$ on the right

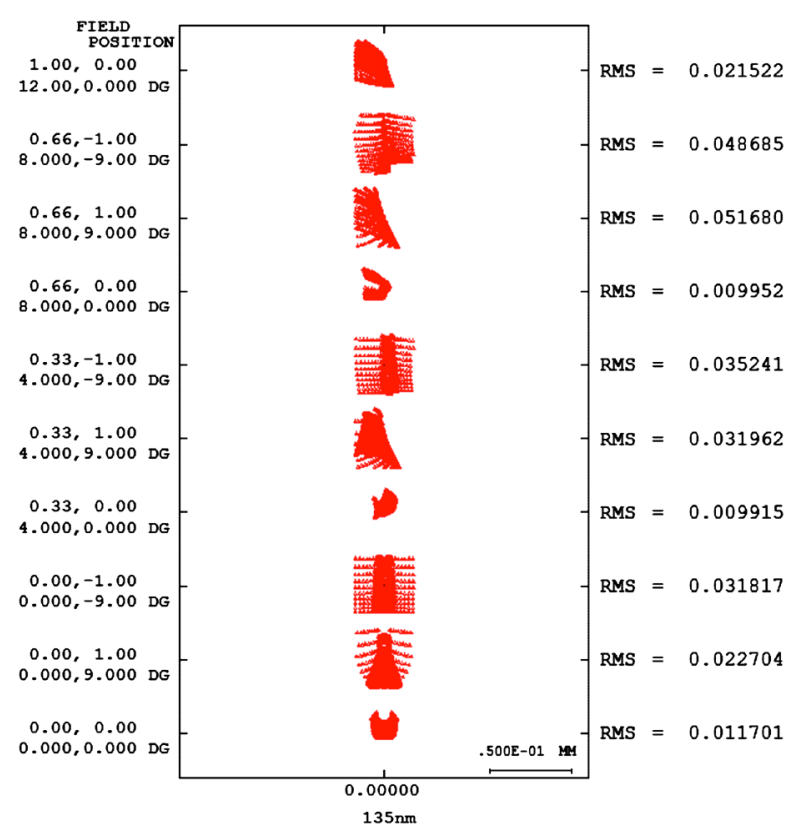

response (black) looks quite complex but after normalization it yields a reasonable profile (blue) with the mean being red shifted by $0.24 \mathrm{~nm}$ and the FWHM is $5.99 \mathrm{~nm}$. The profile shows a high degree of suppression (>99.8\%) of 149.3 and 164.1 lines.

\subsection{Resolution Performance}

As discussed previously the production of the intermediate large image on the grating at a high equivalent $\mathrm{F}$ number is not expected to limit the instrumental performance. The CZT acts mainly as a wavelength filter, and the optimization of the imaging performance depends mainly on focusing the intermediate image on the detectors. Besides M2 there are two mirrors involved in each back imager. Both are off axis conics with additional aspheric coefficients. They have to focus light on the focal planes of the detectors under some serious volume constraints to fit in a small space.

The requirements demand that the spots have $90 \%$ of the encircled energy to fall into $2 \times 2$ resolution elements. A resolution element is an angular region of $0.093^{\circ} \times 0.093^{\circ}$. Note that the 24 degrees vertical FOV divided by 256 pixels is $0.093^{\circ}$. This angle element approximately corresponds to $96 \mu \mathrm{m}$ linear dimension on the detector's photocathode. As in most optical instruments rays with more off-axis positions have more aberrations and as a design goal off axis angles and mirror tilts should be minimized. The design shows that spot sizes reach around $50 \mu \mathrm{m}$ rms maximum and an average around $30 \mu \mathrm{m}$ rms. Figures 21, 22 and Table 4 summarize the different values with fields allocation.

From the above it is evident that the design met the spot size requirements.

The actual resolution performance of the instrument was established from laboratory tests and calibrations performed at the Centre Spatiale de Liege in Belgium. A more detailed account of the functional testing of the instrument is given by Loicq et al. (2016c).

There were 3 vacuum adjustable mirrors M1, SW CM2 and LW CM 2. After extensive alignment in the visible, these mirrors were fine tuned in the vacuum chamber until optimum 
Fig. 22 The same as Fig. 21 except for the LW channel
Table 4 Summary of spot designs for both channels

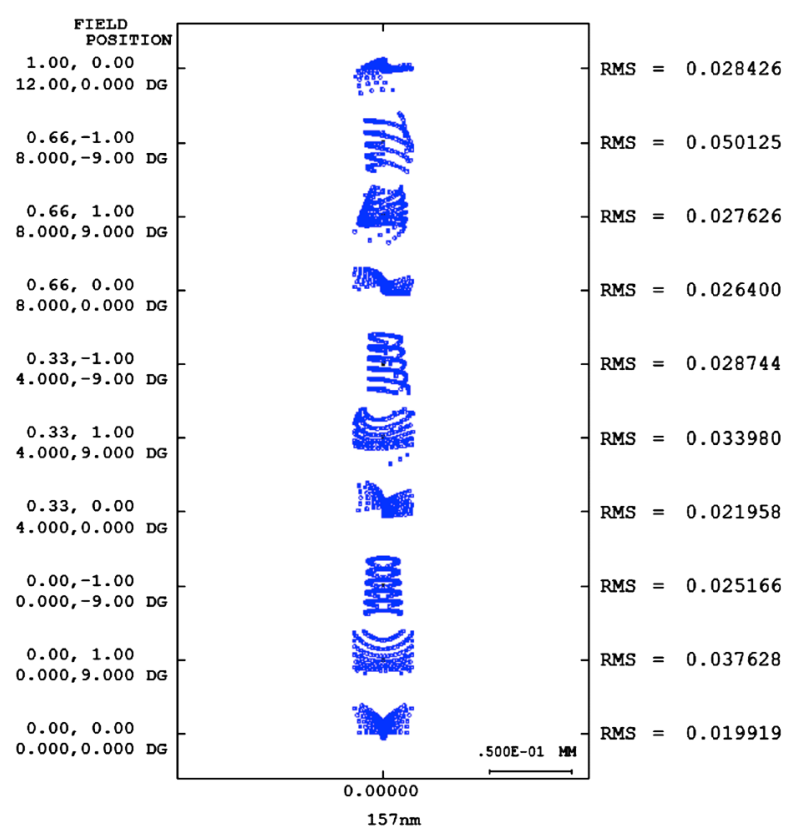

\begin{tabular}{lll}
\hline RMS spot size $(\mu \mathrm{m})$ & SW channel & LW channel \\
\hline Max & 51 & 50 \\
Min & 9 & 19 \\
Average & 27 & 29 \\
\hline
\end{tabular}

spot sizes were achieved. Then the vacuum chamber was opened and the mirror adjustors were clamped according to the procedure discussed previously and staked so that they would not move during vibration tests.

In Figs. 23 and 24 some of the resultant spots are shown after clamping the CM2-s. The numbers in the boxes are the position angles and the percentage of total integrated power in the $2 \times 2$ resolution element box. The net result is that, although the measured spot sizes are not as good as those predicted by the design, the as built instrument satisfies the requirements in most positions in the field.

\subsection{Geometric Distortions}

Due to the back imager off-axis mirrors, distortion is observed in both imagers (SW and LW) with substantial differences in the two imagers. The back imager optics in the SW channel is close to the grating and in this channel the mirrors were placed at a somewhat unfavorable angle causing worse distortion. This was discovered fairly late in the development process and it was decided that the distortion should be corrected in data processing rather than redesigning the instrument layout. The FUV instrument is the largest instrument on ICON, which has to fit between the S/C and the payload deck and configuration changes of FUV would have large perturbations on the S/C design. The distortion patterns for SW and LW are shown in Fig. 25. On both channels the $x$ and $y$ resolutions are different by a factor 


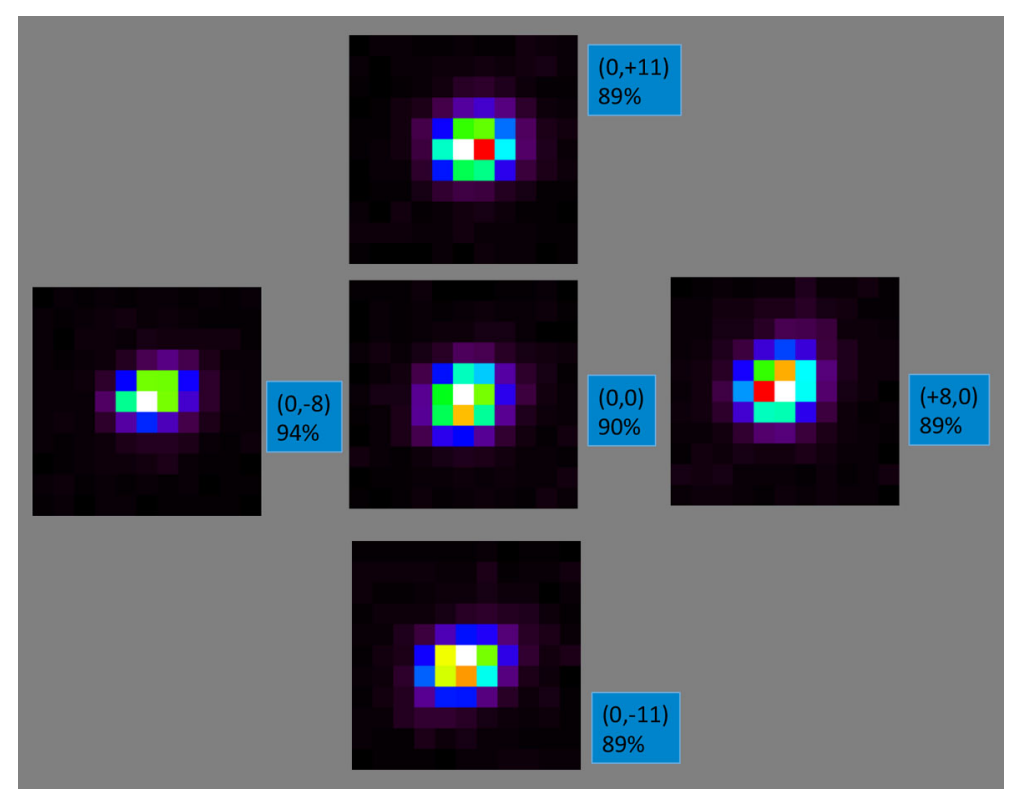

Fig. 23 The SW channel spots at 5 major locations $(-8,0),(0,-11),(0,0),(0,+11)$, and $(+8,0)$ after clamping CM2. The numbers in the box are the position and the percentage of total integrated power in the $2 \times 2$ resolution element box

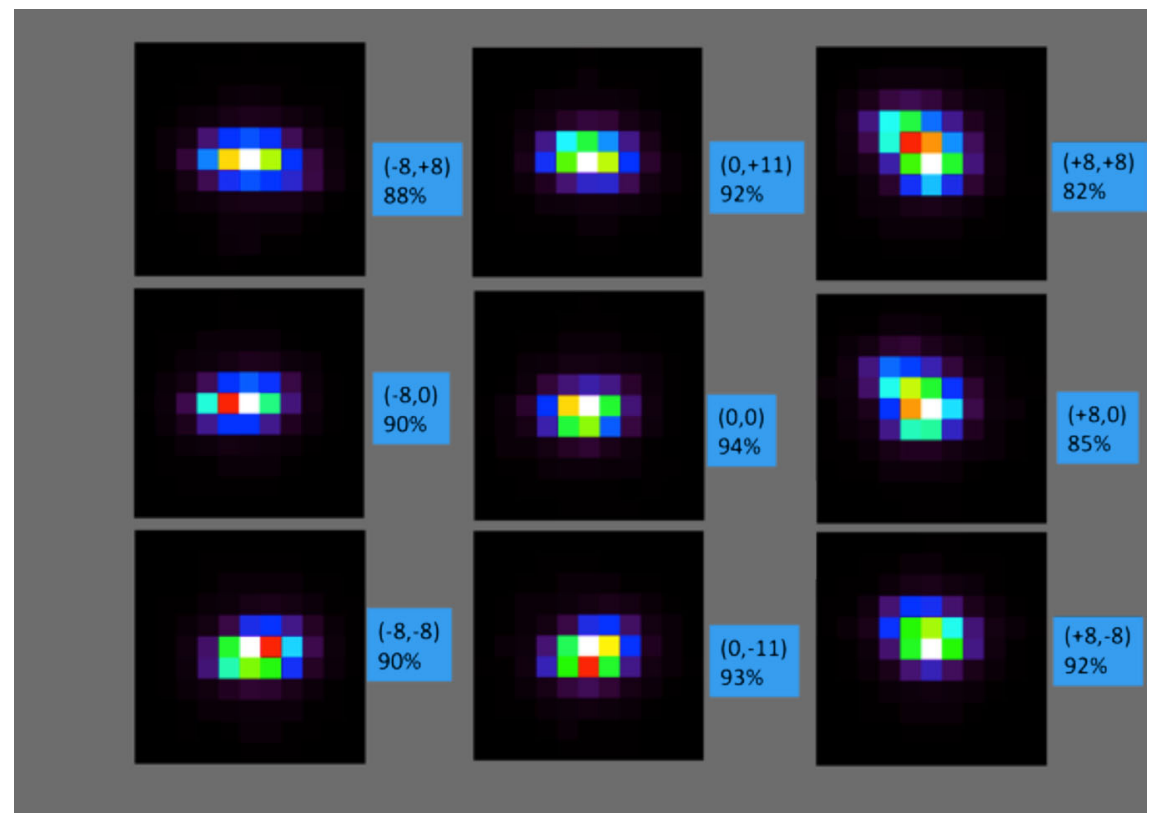

Fig. 24 The LW channel spots at 9 major locations $(-8,+8),(-8,0),(-8,-8),(0,-11),(0,0),(0,+11)$, $(+8,+8),(+8,0)$ and $(+8,-8)$ after clamping CM2. Same as Fig. 23 
Fig. 25 Distortion maps for both channels from the optical model. The effect of distortion is larger on SW channel (left) than LW (right)

Table $5 \mathrm{X}$ and $\mathrm{Y}$ resolution on both channels
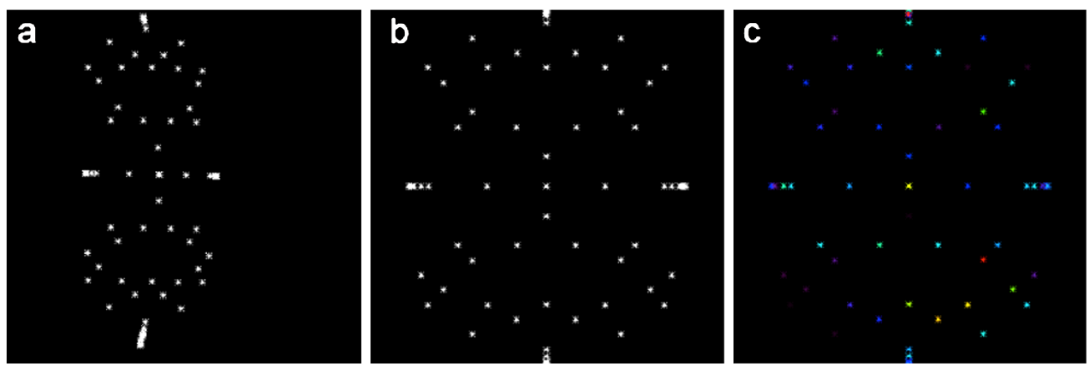

Fig. 26 (a) Each point represents a measurement at a known input angle as set up by the instrument manipulator. (b) Using the distortion correction algorithm the distortion was removed. (c) The same as (b) except the colors show the error from the input angles of each corresponding input beam. Red represent larger errors and blue is the minimum (Color figure online)

of approximately 2 (Table 5). Moreover one can notice the distortion is larger and nonhomogeneous in the SW channel. Aberrations created by the off-axis mirrors (CM1 and CM2) are compensated in LW while they are amplified in the SW channel.

During calibration about $\sim 70$ separate input beam angles were set up with the instrument manipulator and the corresponding $(x, y)$ pixel positions of the spots were recorded. An example of the output data is shown on Fig. 26a. From this data a least squares fit algorithm was developed to be able to predict the output position corresponding to any input angle. Using this algorithm Fig. 26b was produced. This plot should represents the original angles at which the beam had entered the optics. Figure 26c is the same as Fig. 26b except colors are used to represent the error how far off each of these spots are from the input angles of each corresponding input beam. Red represents larger errors and blue is the minimum.

In summary the instrument calibration confirmed all aspects of the design including the instrument's geometric distortion. Distortion correction was always planned as part of the instrument design and distortion correction re-mapping will yield a one resolution cell uncertainty in the final position mapping. 


\subsection{Stray-Light Suppression}

As stated earlier the instrument needs to suppress the $130.4 \mathrm{~nm}$ emission below $2 \%$ of the $135.6 \mathrm{~nm}$ emission in the SW channel. There is also a requirement to minimize Lyman alpha, which is very bright, but significantly further out of band than the $130.4 \mathrm{~nm}$ emission. The rejection of nearest emission features at $149.3 \mathrm{~nm}$ and at $164.1 \mathrm{~nm}$ in the LW channel was also a requirement although these features are much weaker. Another important requirement is to suppress any earthshine or daylight and to make sure that the instrument is essentially solar blind. It is very difficult to test the stray light performance of an instrument in the laboratory. A very large room would be needed with a bright source, which would illuminate the instrument from a known direction while assuring that no spurious reflections from walls would contaminate the results. FRED Optical Engineering Software was used to model the source and the mechanical configuration of the instrument and calculate the stray light performance.

Stray light modeling usually is a two-step process. In step 1, it is assumed that all the light comes from a point source infinitely far away (like a star) and the angle of the direction of the light is varied to express the Point Source Transmission (PST) of the instrument as a function of the angle of incidence. In Step 2, the stray-light source intensities are modeled as a function of the incidence angle at the instrument. This intensity is then combined with the PST and the resultant photon flux is integrated across the whole range of incidence angles. This represents the total stray-light flux the instrument is receiving.

The calculated PSTs are given in Fig. 27 for the SW channel after the first step. The horizontal axis is the radial angle from the optic axis while the azimuth angles are illustrated with the various colored curves in the plot. The vertical axis is the transmitted radiation. The first plot represents the in-band science wavelength at $135.6 \mathrm{~nm}$. At the optic axis the model shows a transmission of about $7 \%$, which is consistent with the number of reflections and the assumed reflective efficiencies. This transmission is constant across the FOV. At larger radial angles as we leave the FOV the transmission drops almost 4 orders of magnitude showing the rejection performance for rays outside the FOV. As we will see most scattering is in fact due to this radiation, which is in band but outside the FOV. The next plot is Lyman alpha and it is always 5 orders of magnitude down inside or outside of the FOV. The rejection is also very effective at $130.4 \mathrm{~nm}$. The last plot is made for visible light at $500 \mathrm{~nm}$ and the stray light rejection performance is shown to be very good here, 8 orders of magnitude without taking into account the solar blindness of the detectors.

The LW channel stray light modeling is shown in Fig. 28. The first set of curves represents the in band light at $157 \mathrm{~nm}$. The second set shows that the design achieved the goal of suppressing the $149.3 \mathrm{~nm}$ atomic nitrogen line. The last set of curves shows that Lyman alpha is also very effectively suppressed in the LW channel.

In order to calculate the total stray light in step 2 the source radiance models were converted to intensity (flux/steradian) by ray tracing. This was accomplished by dividing the atmosphere into a series of earth-centric annular rings corresponding to different altitudes, where each ring is oriented so that it is tangent to the line of sight for a known altitude. For reference the center of the field of view is taken as a tangent altitude of approximately $155 \mathrm{~km}$. Ray powers were computed using the limb radiance and line of sight projections onto the input baffle. The limb radiance model was adopted from the GUVI measurements.

Rays were traced to the aperture and the intensity values were computed on polar coordinate grids that were subsequently used in the source models for the detector irradiance computations. The summary of the computation results is presented in Table 6 . The second row is the signal, which is defined to be the $100 \%$ mark. The third row is the "in band" energy namely the stray light, which is in the instrumental passband but this light is scattered 

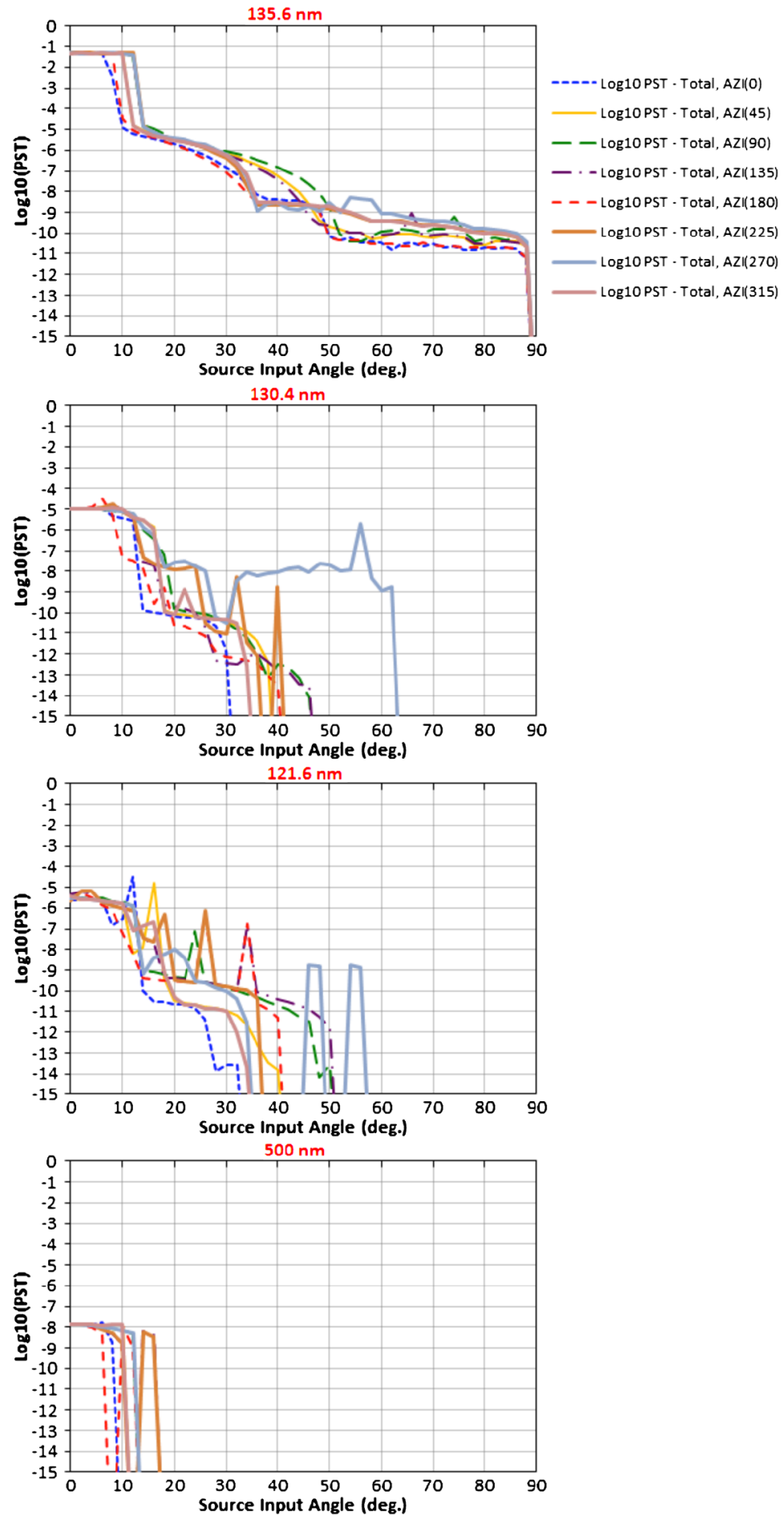

Fig. 27 Stray light rejection modeling for the SW channel 
Fig. 28 Stray light rejection modeling for the LW channel
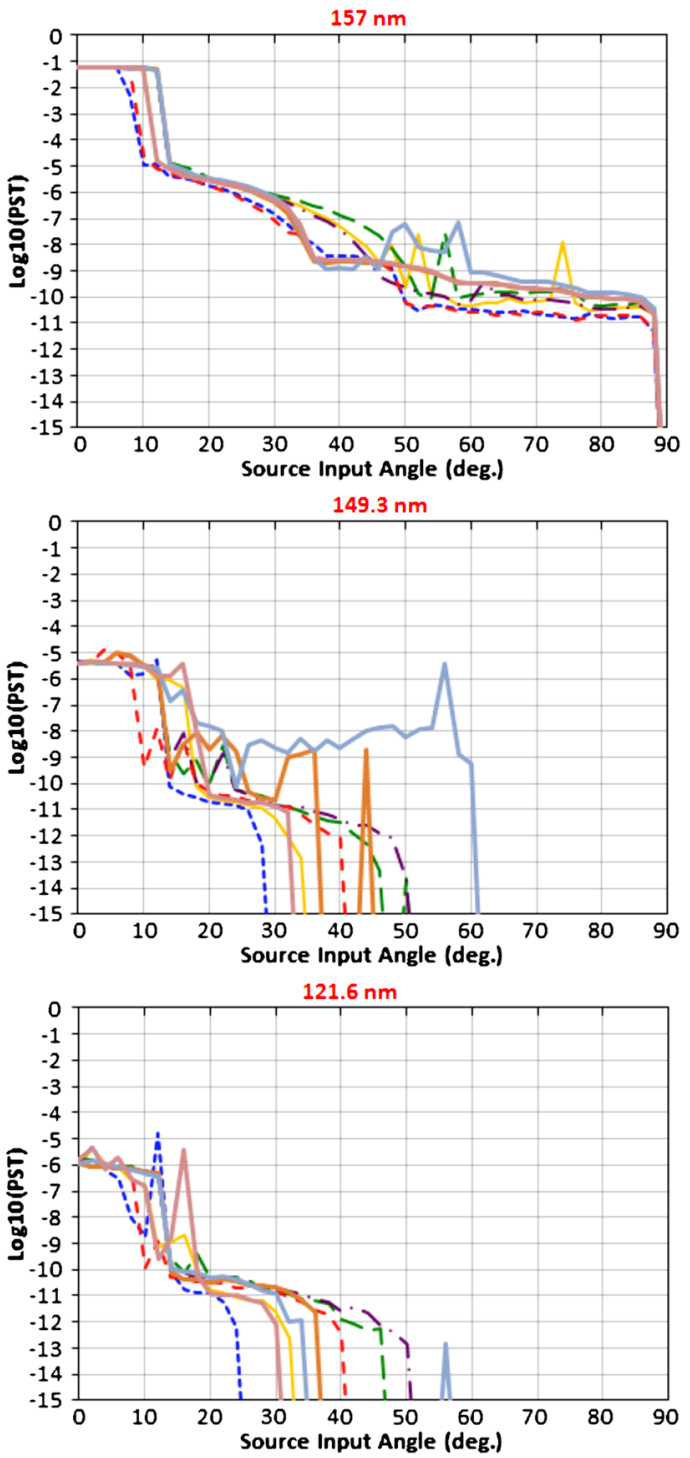

into the instrument even though it originates outside the FOV. The fourth row expresses the third row as a percentage of the second row giving $0.98 \%$ and $0.84 \%$ for the two channels respectively. The 5th row is the component of the scattered light which is in the FOV but which is from spectral regions other than the spectral passband of the instrument. This is the combination of the residual Lyman alpha, $130.4 \mathrm{~nm}$, and all other light from unwanted spectral regions including longer wavelength ultra violet. The numbers in the 5th row and the percentages expressed in the 6th row shows that the dominant stray light contamination is the scattering of the in band radiation from regions outside of the FOV. Nevertheless the combined contamination is still below the $2 \%$ level and therefore ICON FUV meets the stray light requirements. 
S.B. Mende et al.

Table 6 Results of stray light calculations

\begin{tabular}{lll}
\hline & SW Camera & LW camera \\
\hline Signal (photons/s) & $1.51 \mathrm{E}+07$ & $9.42 \mathrm{E}+06$ \\
In band SL (photons/s) & $1.47 \mathrm{E}+05$ & $7.92 \mathrm{E}+04$ \\
In band SL (\%) & $0.98 \%$ & $0.84 \%$ \\
Out of band SL (photons/s) & $4.50 \mathrm{E}+04$ & $3.43 \mathrm{E}+04$ \\
Out of band SL (\%) & $0.30 \%$ & $0.36 \%$ \\
Total SL (\%) & $1.27 \%$ & $1.20 \%$ \\
\hline
\end{tabular}

\subsection{Stray Light Contamination Testing}

The stray light modeling showed that the dominant stray light was due to light in the instrument wavelength pass band originating outside the FOV. This radiation had to be scattered in the instrument to reach the photocathode of the detectors. The other components of unwanted background light are emissions outside of the nominal passband of ICON FUV. Our modeling showed that this component was less important. Both of these stray light contaminations should appear as a diffuse light backgrounds on the detector. Although it was not possible to fully validate the performance of the instrument in rejecting all out of band stray light it was thought important to verify experimentally the instrument's performance in rejecting the key contaminant Lyman alpha (121.6-nm). As it was shown on Fig. 3, limb view in Lyman alpha can be as much as $20 \mathrm{kR}$ during the day pass whereas the emission to be measured at 135.6 OI peaks only at about $10 \mathrm{kR}$. At the same time the rejection of Lyman alpha in the LW channel could also be tested.

During these tests the instrument responsivity at 135.6 was compared to the responsivity for Lyman alpha in the SW channel and it was found to be down by only a factor of 467 . This was considered to be too low Lyman alpha rejection. The modeling showed that the Lyman alpha suppression should be at least four orders of magnitudes. When the GSE monochromator was tuned to another out of band emission at the long wavelength end of FUV to $170 \mathrm{~nm}$, the responsivity in the $135.6 \mathrm{~nm}$ channel was down by a factor of 2140 compared to the referenced 135.6 responsivity. The results implied that the measured rejections were less than the modeled predictions and that stray Lyman alpha might cause some difficulties in interpreting the flight data.

The output of the above stray light tests appeared to be images with the stray light counts concentrated in a relatively small spot. These images were inconsistent with the expected diffuse background scattered inside the instrument as normally seen as stray light contamination. This observation showed that the stray light, that was observed at the output had to be produced by parallel (collimated) light entering at the input aperture of the instrument. The parallel light had to be subsequently diffracted by the flight grating and focused into the observed small spot by the flight instrument. This observation questioned the interpretation of these stray-light tests.

To explore this issue the stray light experiment was repeated but with the inclusion of a $\mathrm{BaF}_{2}$ window in the optical path that completely absorbed all radiation at $121.6 \mathrm{~nm}$. When the test was repeated with the absorber the focused spot was still present proving that the stray light wavelength was not from Lyman alpha. Furthermore the reduction in the counting rates in the image was consistent with transmission of the $\mathrm{BaF}_{2}$ window in the $135.6 \mathrm{~nm}$ wavelength region in the pass-band of the instrument. Although these tests were not $100 \%$ conclusive the apparent "straylight" had to be caused by radiation in the instrument pass band produced by the Optical Ground Support Equipment (OGSE). Our OGSE was a single 
stage monochromator and an off band transmission of $10^{-4}$ would not be unusual for such a monochromator. To account for the observed apparent stray light in the LW channel we would have to invoke "leak" or an off-band transmission of $5 \times 10^{-4}$ or greater for the OGSE monochromator in the wavelength region of the passbands when the OGSE was set to Lyman $\alpha$. Based on these arguments it was decided that the apparent leakage was an artifact due to unwanted light passed by the OGSE.

\subsection{ICON FUV Detectors}

ICON FUV requires modest spatial resolution in its FOV, which can be accomplished by a pixel raster of only $256 \times 256$. ICON detectors have to be sensitive in the spectral ranges around 135.6 and $157 \mathrm{~nm}$ and have the highest quantum efficiency possible consistent with high detectability of each resulting photo-electron. Although photo-conductive detectors like CCDs or CMOS are much simpler and require less resources to operate they could not be used in this application because of their residual sensitivity in the visible and near UV region. ICON has to view the dayside atmosphere and having a highly "solar blind" detector is of paramount importance. All of these requirements favor photo-emissive detectors with MCP electron amplification in the same manner as it was required for the IMAGE FUV instrument to view the aurora in the day-lit atmosphere. There are several ways to detect the FUV generated photo electron clouds from an MCP.

Dayglow limb emissions in the FUV can be quite intense, several kiloRayleighs, and can fill the entire FOV producing total counting rates that exceed the capabilities of cross delay line type detectors as used in the IMAGE SI instrument (Mende et al. 2000c). To accommodate the large dynamic range we chose the type of detector, which was used in the IMAGE Wideband Imaging Camera (WIC), consisting of a photo-cathode on an MCP, an output phosphor, and a fiber-optics taper to couple the light from the phosphor to a CCD (Mende et al. 2000b). ICON FUV optics produces a flat image unlike WIC so the ICON detector was considerably simpler. In fact the front end was quite similar to a $25 \mathrm{~mm}$ conventional intensifier tube (Fig. 29). The ICON intensifier has a dual stack MCP to provide enough gain to detect phosphor scintillations due to single photo-electron emission at the photocathode. One significant departure from the IMAGE WIC detector was the use of completely sealed

Fig. 29 ICON FUV converter tube

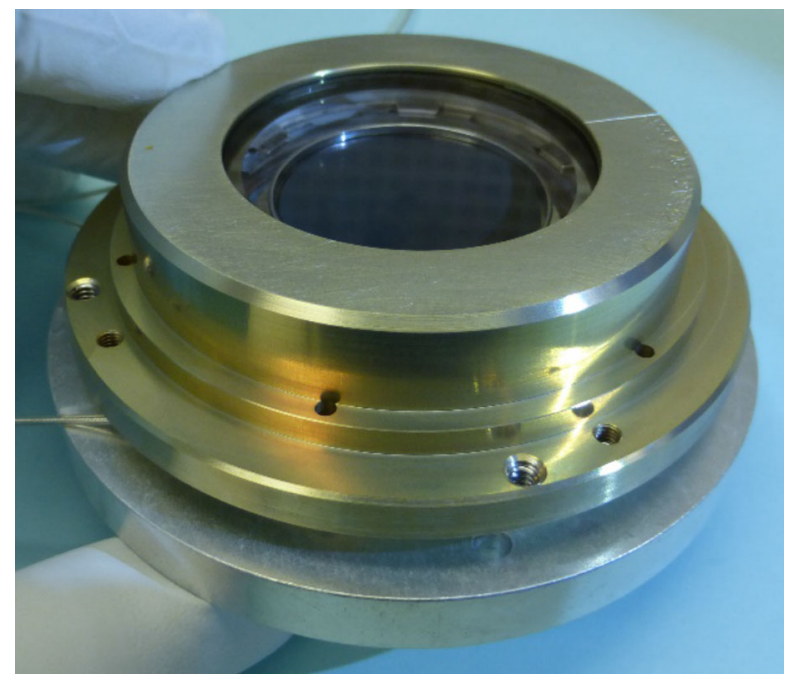


Fig. 30 ICON flight camera shown integrated with the FUV image converter

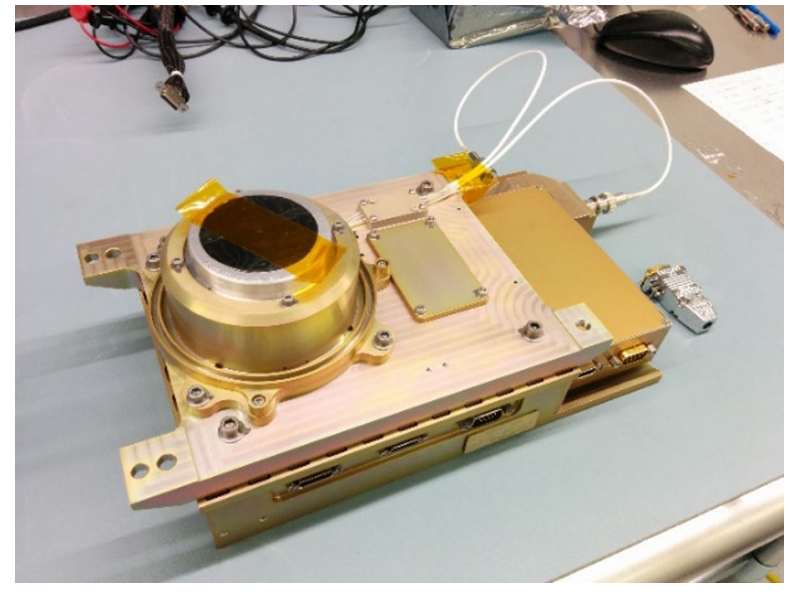

tubes on ICON instead of the vented detectors of WIC. It was thought that the sealed tubes harbored less contamination risk during ground processing and allowed testing the entire detector chain without having to evacuate the detector.

In some ways the ICON detectors were also very similar to the detectors used in the spacecraft experiment named Imager of Sprites and Upper Atmospheric Lightning (ISUAL) designed and built at the Space Science Laboratory at UC Berkeley. This instrument had a camera with a $25 \mathrm{~mm}$ image tube that was fiber-optically coupled to a frame transfer CCD. These devices were space qualified including tests of their radiation tolerance to energetic proton bombardment. In ICON the same Teledyne DALSA FTT1010M $1024 \times 1024$ frame transfer CCDs were used. The CCD-s were binned on the chip $2 \times 2$ so the "native" readout provided a $512 \times 512$ image from the cameras.

The FUV converter tubes were built and characterized and their photocathode quantum efficiency was measured in Berkeley. The complete converters were integrated into the camera at Space Dynamics Laboratory (SDL) of the Utah State University in Logan Utah. The process included bonding the converters to a fiber-optic taper and to the readout CCD. SDL assembled and tested the complete cameras (Champagne 2015), except the high voltage power supplies for the intensifiers, which were built by UC Berkeley. A completed camera is shown on Fig. 30.

The camera readout electronics provides video imagery to the ICP over a 21 bit serialized interface, nominally at 10 frames per second and in $512 \times 512$ format $(2 \times 2$ pixel binning on chip). During science operations the pixels are further binned $2 \times 2$ by the processing electronics therefore the $1024 \times 1024 \mathrm{CCD}$ pixels are binned to $256 \times 256$. The CCD and primary electronics assembly reside in separate thermal zones, to minimize dark current without active cooling. The CCD cameras were fully characterized with both visible light (prior to integration with the UV converter) and UV photons (following system integration). Measured parameters included camera dark current, dark signal non-uniformity, read noise, linearity, gain, pulse height distribution, dynamic range, charge transfer efficiency, resolution, relative efficiency, quantum efficiency, and full well capacity. UV characterization of the camera systems over a range of micro-channel plate (MCP) voltages during thermal vacuum testing demonstrated that camera performance met the critical on-orbit FUV dynamic range requirements. 


\subsection{Detector Performance}

As we have seen in Sect. 3.1 as (4), a generalized instrument converts $P_{0}$ photons into $P_{1}$ information quanta with a conversion efficiency of $\varepsilon$ :

$$
P_{1}=P_{0} \varepsilon=P_{0} T q_{e}
$$

where $P_{0}$ is the mean number of incident FUV photons and $P_{1}$ is the mean number of photo-electrons produced during the exposure time.and $\varepsilon$ is the overall efficiency. $\varepsilon$ is the combination of $q_{e}$, the quantum efficiency and the optical efficiency $T$, of the reflections and transmissions of the optical components.

In an efficient detector system the signal to noise ratio should approach the intrinsic Poissonian fluctuation noise and additional noise components should be minimal. We have chosen a double MCP system that has a large gain and produces a large electron shower to make an intense light pulses in the phosphor for each photo-electron. This light is detected by the CCD and converted into a large electrical signal $P_{2}$. As long as this gain is large, several of the additional downstream noise sources such as the CCD dark current and readout noise become negligible and the signal to noise ratio $P_{2} / \nabla P_{2}$ is similar to $P_{1} / \nabla P_{1}$. In this section we show that our readout system is highly efficient and will reproduce approximately the SNR of the photo electrons albeit with a slight impairment on account of the MCP multiplication noise.

The signal at the output of the CCD is:

$$
\overline{P_{2}}=\overline{P_{1}} \bar{g}
$$

where $\bar{g}$ is the mean gain of the image tube combined with the mean photon conversion gain of the intensified CCD or the ratio of CCD output electrons per primary photo electron in the image tube. Since the multiplication gain, $g$ also has a statistical distribution additional noise is generated by the multiplication statistics and the mean square fluctuations in CCD output signal, $P_{2}$, is given by (Mandel 1959)

$$
\overline{\nabla^{2} P_{2}}=\overline{\nabla^{2} P_{1}} \bar{g}^{2}+\overline{\nabla^{2} g} P_{1}
$$

But for any distribution the means square deviation (noise)is the same as the mean square minus the square of the mean of the distribution and

$$
\overline{\nabla^{2} g}=\overline{g^{2}}-\bar{g}^{2}
$$

Therefore

$$
\overline{\nabla^{2} P_{2}}=\overline{\nabla^{2} P_{1}} \bar{g}^{2}+\left(\overline{g^{2}}-\bar{g}^{2}\right) \bar{P}_{1}=\left(\overline{\nabla^{2} P_{1}}-\bar{P}_{1}\right) \bar{g}^{2}+\bar{g}^{2} \bar{P}_{1}
$$

and since the distribution of $P_{1}$ is Poissonian

$$
\left(\overline{\nabla^{2} P_{1}}-\bar{P}_{1}\right)=0
$$

we have

$$
\overline{\nabla^{2} P_{2}}=\overline{g^{2}} \bar{P}_{1}
$$

In summary there is an increase in the noise due to the random distribution of the multiplication gain and the resultant mean square fluctuation (noise squared) is equal to the mean photoelectron counts multiplied by the mean square of the gain distribution.

This formulation allows us to formulate the signal to noise ratio at the output of the $\mathrm{CCD}$. We calculate the signal in a science pixel which is equivalent to an angular region of $0.093^{\circ}$ (vertical) $\times 3.0^{\circ}$ (horizontal) approximately equal to $1 \times 32$ resolution elements 
Fig. 31 Pulse height distribution (blue) when the camera is operated with an MCP high voltage of 2350 Volts. DN-s are digitization units of the CCD's A-D converter where $16 \mathrm{CCD}$ electrons (not to confuse with MCP electrons) are $1 \mathrm{DN}$ unit

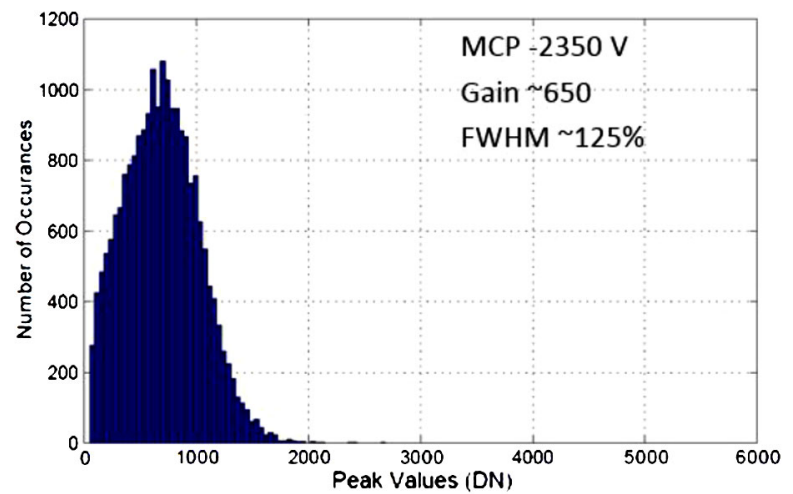

during a 12 second exposure. Using the formulae above, we sum four noise sources, mean square fluctuations of the signal from equation (5) the signal from stray background photoelectron from the photocathode, the CCD readout noise $\mathrm{N}_{\mathrm{r}}$ electrons and the CCD dark current integrated in during the exposure time.

The signal to noise ratio (SNR) is given by the formula

$$
S N R=\frac{P_{1} \bar{g}}{\sqrt{P_{1} \overline{g^{2}}+I_{p} \overline{g^{2}}+\sum N_{r}^{2}+\sum N_{d c}}}
$$

where $P_{1}=$ signal in photoelectron counts in the area collected during exposure time, $g=$ gain of the intensifier in CCD electrons/photoelectron, $I_{p}=$ stray light induced photoelectrons, $N_{r}=$ rms readout noise (electrons), $N_{d c}=$ dark current of CCD of the CCD integrated during the exposure. The unit is electrons which is $1 / 16$ th of the A-D unit in the camera.

We have obtained the mean and the root mean squared gain as 650 and 734 respectively from the distribution shown in Fig. 31. Note that the first two terms at the bottom in (6) are proportional to the mean square of the gain. If there were no spread in the gain and every photo-electron experienced the same gain then the terms should have been the just square of the noise which is the product of the mean gain and the fluctuation in the photoelectrons which is the square root of $P$. Including the multiplication noise the multiplier grows from the square of the mean to the mean square of the distribution.

In calculating the SNR the terms at the bottom representing the image tube dark count $I_{p}$ is less than 10 photoelectron counts per sec per entire detector face, its per pixel contribution is negligible. The other terms containing $N_{r}$ and $N_{d c}$ are also negligible when we recognize that the mean gain, $g$ is $650 \times 16=10400$ converting photo-electrons to CCD-electrons. Thus the SNR is impaired because the formula for the signal (top) is amplified by the mean intensifier gain, $g=650 \times 16$ whereas the photo-electron noise at the bottom is amplified by the root mean square of the intensifier gain $=734 \times 16$. In summary the resultant SNR is less by a factor of $650 / 734$ or $90 \%$ of the SNR estimate of photoelectron counting with noiseless multiplication. The same SNR impairment would result from a photoelectrons reduction equal to the square of the $90 \%$ i.e. $81 \%$. In Table 8 we included this as the equivalent reduction in photoelectron counts to calculate the margin in responsivity to the requirements.

\section{ICON FUV Instrument Performance}

The responsivity of all imaging instruments is the combination of two major factors (1) the number of photons collected, $P_{0}$ defined by the input aperture area and the solid angle of the 
field of view (2) $\varepsilon$, the overall conversion efficiency, the efficiency at which input photons are converted into measurable output signal. Measurable signal is defined by the signal to noise ratio (SNR). In an idealized Poissonian system, for example when photo-electrons are emitted by a photocathode, the SNR is equal to the square root of the number of collected photo-electrons. The SNR performance of any system can be characterized by the number of equivalent output photo-electrons which is equal to the square of the SNR at the output. Thus the overall conversion efficiency $\varepsilon$, is the ratio ratio of the equivalent output photoelectrons (SNR squared) divided by the number of input photons. ICON FUV underwent extensive calibration and the conversion efficiency was measured and the responsivity was estimated by combining the efficiency with the geometrically measured FOV and aperture size at the input slit.

To evaluate the ICON FUV imager performance we have defined several convenient spatial bins. A resolution element is an angular bin of $0.093^{\circ} \times 0.093^{\circ}$ and this corresponds to 1 binned pixel on the detector. Note that the geometric distortion does not allow linear transformation of the detector pixels to angular sampling elements. There are $256 \times 256$ binned pixels on the detector. The vertical dimension of $0.093^{\circ}$ is derived from the $24^{\circ}$ vertical dimension of the FOV divided into 256 binned pixels. The $0.093^{\circ}$ angle also represents a $\sim 4 \mathrm{~km}$ altitude step at a tangent height of $155 \mathrm{~km}$.

In Table 7 we have listed the most significant characteristics of the ICON FUV instrument. For the sensitivity calculations we assumed that the instrument field of view was divided into 6 vertical stripes (line 4 Table 7). The science pixel field of view is therefore $3.0^{\circ}$ wide and one VRE or $0.093^{\circ}$ tall (line 7). Combining this with the measured input slit dimension of $5 \times 32 \mathrm{~mm}$ provides the etendue per science pixel (Table 7 line 11) and the photon collection rate (line 12).

The overall efficiency of ICON FUV was measured directly in the lab during calibrations at CSL. In Table 8 we list the various components and their transmissions for each channel. The predicted efficiency of the system, line 5 is the combination of reflectivity of the mirrors and the grating and the photocathode efficiency. The calculated efficiency is somewhat smaller than $1 \%$. Unfortunately the measured value obtained from the calibration (line 6) is smaller than the predicted value. For the SW channel this discrepancy is about a factor of 2 lower but it is substantially more different for the LW channel. The cause for this discrepancy is unknown. In line 7 we tabulated the predicted instrumental counting rates for the two channels and in line 8 the actual measured calibrated counting rates. The science requirement was written in terms of counting rate per altitude bins regardless of the horizontal size of the bin and it is shown in line 9. The counting rate is given here for one stripe out of six and several stripes can be co-added to improve the signal to noise ratio and meet the science requirement with more margin.

The data presented in Table 8 shows 97.5 predicted and 49 measured counts/s/kR for the $135.6 \mathrm{~nm}$ SW channel per science pixel. There are 6 parallel vertical stripes and the data could be summed up from the six adjacent strips. However the outermost strips have FOV limitations at the top and at the bottom due to the oval shape of the FOV.

It is interesting to compare responsivities with previously flown FUV imagers. IMAGE SI $135.6 \mathrm{~nm}$ channel provides a ready example for comparison. A resolution element is defined as a region of $0.093 \times 0.093$ degree region. There are 192 resolution elements across the 6 channels representing $192 / 6=32$ horizontal resolution elements. The counting rate would be $49.0 / 32=1.5$ counts $/ \mathrm{s} / \mathrm{kR}$ per single resolution element. The IMAGE SI had a $128 \times 128$ pixel raster and a counting rate of 3 counts per $\mathrm{kR} / \mathrm{s}$ in its $135.6 \mathrm{~nm}$ channel. If its image were subdivided to a $256 \times 256$ pixel raster like in ICON then its counting rate would be 0.75 counts $/ \mathrm{s} / \mathrm{kR}$. We consider that this is in fair agreement with the ICON numbers. We had hoped for better performance because the ICON camera has a larger FOV and 
Table 7 ICON FUV performance summary

\begin{tabular}{|c|c|c|c|}
\hline & Parameter & Value & Remarks \\
\hline 1 & Orbit & $\begin{array}{l}575 \mathrm{~km} \text { circular } 27 \\
\text { degree inclination }\end{array}$ & Launch from Kwajalein \\
\hline 2 & Optic axis & $\begin{array}{l}\text { Perpendicular to velocity } \\
\text { vector north. } 20^{\circ} \text { below } \\
\text { local horizontal. }\end{array}$ & Steering mirror allows $\pm 30^{\circ}$ \\
\hline 3 & Field of View & $\begin{array}{l}24^{\circ} \times 18^{\circ}(\text { vertical } \times \\
\text { horizontal })\end{array}$ & \\
\hline 4 & $\begin{array}{l}\text { Number of science } \\
\text { pixels }^{\mathrm{a}} \text { in vertical stripes }\end{array}$ & $\begin{array}{l}\sim 256 \times 6(\text { vertical } \times \\
\text { horizontal })\end{array}$ & For limb brightness profile \\
\hline 5 & $\begin{array}{l}\text { Number of pixels for } \\
\text { imaging }\end{array}$ & $\begin{array}{l}256 \times 192(\text { vertical } \times \\
\text { horizontal })\end{array}$ & TDI imaging \\
\hline 6 & $\begin{array}{l}\text { Maximum altitude } \\
\text { coverage }\end{array}$ & $-8^{\circ}$ to $-32^{\circ}$ elevation & $-8^{\circ}=$ tangent height of $507 \mathrm{~km}$ \\
\hline 7 & $\begin{array}{l}\text { Pixel FOV for vertical } \\
\text { stripe }\end{array}$ & $3.0 \times 0.093^{\circ}$ & 6 vertical stripes \\
\hline 8 & $\begin{array}{l}\text { Pixel FOV for 2di } \\
\text { imaging }\end{array}$ & $0.09375^{\circ} \times 0.09375^{\circ}$ & TDI imaging \\
\hline 9 & Slit area $5 \mathrm{~mm} \times 32 \mathrm{~mm}$ & 1.6 & $\mathrm{~cm}^{2}$ \\
\hline 10 & Field of view $24^{\circ} \times 18^{\circ}$ & $\begin{array}{l}0.14 \text { (truncated circle) } \\
\text { equivalent F No. }=2.7\end{array}$ & $\mathrm{sr}$ \\
\hline 11 & Étendue per science pixel & $1.3 \mathrm{e}-04 \mathrm{~cm}^{2} \mathrm{sr}$ & $\Omega=0.093 \times 3.0$ degrees \\
\hline 12 & $\begin{array}{l}\text { Geometric photon } \\
\text { collection rate at input } \\
\text { aperture }\end{array}$ & 10.9 & Photon/s/science pixel/Rayleigh \\
\hline
\end{tabular}

a Science pixel: $\Omega=0.093^{\circ}$ (vertical) $\times 3.0^{\circ}$ (horizontal) $\rightarrow 0.093^{\circ}$ corresponds to a Vertical Resolution Element (VRE) of $4 \mathrm{~km}$ altitude at tangent height of $155 \mathrm{~km}=\sim 1$ binned pixel $=4 \mathrm{CCD}$ pixels

therefore larger etendue and it does not have a 1/3rd transmission grill in its entrance slit. Unfortunately the lower grating efficiency and the relatively low detector quantum efficiency cancelled some of the advantages the ICON instrument should have had.

The LW channel measurements do not agree well with predicted performance. The most likely explanation of this is the (difficult) grating efficiency measurement. The grating efficiency in the SW is twice that in the LW; this is difficult to understand on physical grounds given the relatively small difference in wavelength. If we assumed the two grating efficiencies were equal, as would be expected from analytical modeling, then the LW channel efficiency is closer to that predicted and the differences can be attributed to variations in reflectivity optic to optic, whis is a particularly important factor in a multi-reflection system like ICON FUV.

\section{Summary}

ICON FUV fulfills the requirement of making remote sensing measurements of the $\mathrm{O}$ to $\mathrm{N}_{2}$ composition of the sunlit atmosphere and $\mathrm{O}^{+}$density at nighttime. To make these measurements successfully, the instrument has to satisfy several requirements of high performance in spatial and temporal resolution and of responsivity. The responsivity depends on the photon collection efficiency at the input aperture and on the efficiency of conversion of photons 
Table 8 ICON responsivity by channel

\begin{tabular}{lll}
\hline & SW & LW \\
\hline Mirror reflective efficiency & $46.42 \%$ & $38.95 \%$ \\
Grating efficiency & 0.175 & 0.3 \\
Quantum efficiency detector & 0.11 & 0.07 \\
Modeled efficiency of system & $0.89 \%$ & $0.82 \%$ \\
Measured efficiency of system & $0.45 \%$ & $0.16 \%$ \\
Counting rate/science pixel/s/kR & 97.5 & 89.2 \\
Measured counting rate/science pixel/s/kR & 49 a & $17.4{ }^{\mathrm{a}}$ \\
Multiplication and CCD noise equivalent reduction & $81 \%$ & $81 \%$ \\
Measured responsivity/science pixel/s/kR & 39.7 & 14.1 \\
Responsivity requirement from Sect. 2.3 (counting rate/science pixel/s/kR) & 13 & 8.3 \\
Responsivity margin & $205 \%$ & $70 \%$ \\
Counts during nominal 12 s exposure (counts/Rayleigh/science pixel) & 0.48 & 0.17 \\
\hline
\end{tabular}

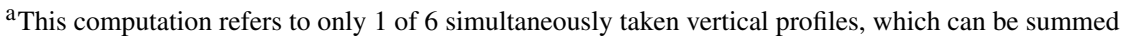

into measureable output signal. The ICON FUV instrument is a spectrographic imager producing spectrally filtered two dimensional FUV images in two wavelength bands. When the atmosphere is sunlit ICON FUV will measure the limb altitude profile of two atmospheric emissions and obtain vertical profiles of the atmospheric $\mathrm{O} / \mathrm{N}_{2}$ ratio. When the atmosphere is in the dark ICON FUV will measure the limb profile of the light emitted at $135.6 \mathrm{~nm}$ produced by the recombination of $\mathrm{O}^{+}$ions. This measurement permits the computation of the nighttime ionospheric density altitude distribution. During these conditions the ionospheric $135.6 \mathrm{~nm}$ data will be processed as altitude distributions and in addition the data will be presented in the form of longitude and latitude distribution primarily to obtain two dimensional images of equatorial spread-F ion distributions. ICON FUV will be viewing essentially perpendicular to the orbit velocity vector and vertically downward with its optic axis $20^{\circ}$ below local horizontal. ICON has a steering mirror that allows the FOV to be steered horizontally up to $30^{\circ}$ forward and aft from its nominal position perpendicular to the velocity vector. This will enable ICON FUV to look along the magnetic meridian for magnetic field aligned ionization regions.

The instrument is based on the Czerny-Turner design with two concave spherical mirrors and a flat grating. The first, the collimator mirror, is relatively small while the second, the camera mirror, is large. There are two exit slits corresponding to the two wavelength channels and two back imager cameras one for each channel to produce images on two detectors. The detectors are micro channel plate (MCP) intensified FUV tubes with phosphor coupled to CCD-s to convert the light pulses to electrical signals. The MCP-s are dual stack to amplify the signal so that the sunbsequently added CCD and other noises will be negligible. The CCD-s will be scanned at 8.3 frames per second and 100 frames will be co-added to digitally create 12 second integrated images for data down linking. This will cover all the dynamic range encountered in day and night FUV airglow. The limited downlink requires extensive data processing on board to calculate the 12 second exposure limb altitude profiles and the two dimensional longitude-latitude distributions of the ionization. These computations are performed in real time using look up tables in the FPGA-s resident in the ICON Instrument Control Processor (ICP) Unit. ICON FUV was constructed in the United States. It was first aligned in the visible wavelength region by using a grating whose ruling density 
was exactly one fourth of the flight grating's ruling density and by using visible CCD cameras. The instrument was then shipped to the Centre Spatiale de Liege (CSL) in Belgium where a special GSE calibration system was constructed to perform the vacuum ultraviolet testing and calibration of ICON FUV. The instrument alignment was fine-tuned at the working FUV wavelength and the instrument was finalized for environmental testing. It was tested and calibrated at CSL and was shipped back to the US for payload integration. The tests showed that the instrument met its requirements including its resolution requirements in its final configuration. Stray light rejection properties of the instrument were investigated by modeling and by vacuum chamber tests and it was shown that stray light suppression is adequate. It was known from the design and was verified in the laboratory that there is significant geometric distortion in the optics. The data processing system is designed to compensate for this distortion. The sensitivity of the instrument was estimated using measurements made during calibrations at CSL. This showed that although the sensitivity of the instrument is not as high as it was originally envisaged it still has positive margin when compared to the scientific requirements as they had been defined.

Acknowledgements The authors acknowledge the contributions of many persons who made it possible for us to build the ICON FUV experiment. There are too many persons to be named individually at the various institutions which cooperated in producing the ICON FUV instrument at the University of California, Berkeley, at the Centre Spatiale de Liege in Belgium, at the Space Dynamics Laboratory in Logan Utah, and the Lockheed Advanced Technology Center in Palo Alto, at the Goddard Spaceflight Center in Greenbelt Maryland, at Orbital-ATK in Dulles, Virginia and at several other institutions. The authors gratefully acknowledge funding by the NASA's Explorers Program under the Ionospheric CONnection Explorer (ICON) project contract number NNG12FA45C.

\section{References}

C.D. Anger, S.K. Babey, A.L. Broadfoot, R.G. Brown, L.L. Cogger, R. Gattinger, J.W. Haslett, R.A. King, D.J. McEwen, J.S. Murphree, E.H. Richardson, B.R. Sandel, K. Smith, A.V. Jones, An ultraviolet auroral imager for the Viking spacecraft. Geophys. Res. Lett. 14, 387 (1987)

D.R. Austin, T. Witting, I.A. Walmsley, Broadband astigmatism-free Czerny-Turner imaging spectrometer using spherical mirrors. Appl. Opt. 48, 3846 (2009). doi:10.1364/AO.48.003846

C.A. Barth, S. Schaffner, Ogo4 spectrometer measurements of the tropical ultraviolet airglow. J. Geophys. Res. 75, 4299-4306 (1970)

B. Bates, M. McDowell, A.C. Newton, Correction of astigmatism in a Czerny-Turner spectrograph using a plane grating in divergent illumination. J. Phys. E, Sci. Instrum. 3, 206-210 (1970)

P. Blain, R. Desselle, I. Domken, C. Kintziger, E. Renotte, Y.G. Stockman, C. Chou, H.U. Frey, K. Rider, S.B. Mende, J.J.D. Loicq, VUV optical ground system equipment and its application to the ICON FUV flight grating characterization and selection. Proc. SPIE 9912, 991240 (2016)

J. Champagne, Design and characterization of the CCD detector assemblies for ICON FUV, in AGU Fall Meeting (AGU, Washington, 2015)

A.B. Christensen, L.J. Paxton, S. Avery et al., Initial observations with the Global Ultraviolet Imager (GUVI) in the NASA TIMED satellite mission. J. Geophys. Res. 108(A12), 1451 (2003). doi:10.1029/ 2003JA009918

A.B. Christensen, L.J. Paxton, S. Avery, J. Craven, G. Crowley, D.C. Humm, H. Kil, R.R. Meier, C.-I. Meng, D. Morrison, B.S. Ogorzalek, P. Straus, D.J. Strickland, R.M. Swenson, R.L. Walterscheid, B. Wolven, Y. Zhang, Initial observations with the Global Ultraviolet Imager (GUVI) in the NASA TIMED satellite mission. J. Geophys. Res. 108 (SIA 16-1), 1451 (2003). doi:10.1029/2003JA009918

J. Comberiate, F. Kamalabadi, L.J. Paxton, A tomographic model for ionospheric imaging with the Global Ultraviolet Imager. Radio Sci. 42(2), RS2011 (2007). doi:10.1029/2005RS003348

M. Czerny, A. Turner, Über den Astigmatismus bei Spiegelspektrometern. Z. Phys. A 61, $792-797$ (1930)

M.L. Dalton, Astigmatism compensation in the Czerny-Turner spectrometer. Appl. Opt. 5, 1121-1123 (1966). doi:10.1364/AO.5.001121

L.A. Frank, J.D. Craven, Imaging results from dynamics explorer 1. Rev. Geophys. 2, 249 (1988)

L.A. Frank, J.D. Craven, K.L. Ackerson, M.R. English, R.H. Eather, R.L. Carovillano, Global auroral imaging instrumentation for the dynamics explorer mission. Space Sci. Instrum. 5, 369-393 (1981) 
W.B. Hanson, A comparison of the oxygen ion-ion neutralization and radiative recombination mechanisms for producing the ultraviolet nightglow. J. Geophys. Res. 75, 4343-4346 (1970). doi:10.1029/ JA075i022p04343

G.T. Hicks, T.A. Chubb, Equatorial aurora/airglow in the far ultra-violet. J. Geophys. Res. 75, 6233-6248 (1970)

J.D. Huba, K.F. Dymond, G. Joyce, A.A. Budzien, S.E. Thonnard, J.A. Fedder, R.P. McCoy, Comparison of O+demsity from ARGOSLORAAS data analysis and SAMI2 model results. Geophys. Res. Lett. 29 (2002). doi:10.1029/2001GL013089

T.J. Immel, S.L. England, S.B. Mende, R.A. Heelis, C.R. Englert, J. Edelstein, H.U. Frey, E.R. Taylor, W.W. Craig, S.E. Harris, M. Bester, G.S. Bust, G. Crowley, J.M. Forbes, J.-C. Gèrard, J.M. Harlander, J.D. Huba, B. Hubert, F. Kamalabadi, J.J. Makela, A.I. Maute, R.R. Meier, C. Raftery, P. Rochus, O.H.W. Siegmund, A.W. Stephan, G.R. Swenson, S. Frey, D.L. Hysell, A. Saito, K.A. Rider, M.M. Sirk, M.H. Stevens, The ionospheric connection explorer mission: mission goals and design. Space Sci. Rev. (2017)

T.J. Immel, E. Sagawa, S.L. England, S.B. Henderson, M.E. Hagan, S.B. Mende, H.U. Frey, C.M. Swenson, L.J. Paxton, Control of equatorial ionospheric morphology by atmospheric tides. Geophys. Res. Lett. 33, L15108 (2006). doi:10.1029/2006GL026161

F. Kamalabadi, J.M. Comberiate, M.J. Taylor, P.-D. Pautet, Estimation of electron densities in the lower thermosphere from GUVI $135.6 \mathrm{~nm}$ tomographic inversions in support of SpreadFEx. Ann. Geophys. 27, 2439-2448 (2009). doi:10.5194/angeo-27-2439-2009

F. Kamalabadi, J. Qin, B. Harding, D. Iliou, J. Makela, R.R. Meier, S.L. England, H.U. Frey, S.B. Mende, T.J. Immel, Inferring nighttime ionospheric parameters with the far ultraviolet imager onboard the ionospheric connection explorer. Space Sci. Rev. (2017)

J. Loicq, C. Kintziger, A. Mazzoli, T. Miller, C. Chou, T. Immel, H. Frey, S. Mende, Optical design and optical properties of a VUV spectrographic imager for ICON mission. Proc. SPIE 9905, 990507 (2016a)

J. Loicq, P. Blain, R. Desselle, I. Domken, C. Kintziger, E. Renotte, Y. Stockman, C. Chou, H. Frey, K. Rider, $\mathrm{S}$. Mende, VUV optical ground system equipment and its application to the ICON-FUV flight grating characterization and selection. Proc. SPIE 9912, 991240 (2016b)

J. Loicq, P. Blain, R. Desselle, I. Domken, C. Kintziger, E. Renotte, Y. Stockman, L. Clermont, C. Chou, C. Popette, H. Frey, K. Rider, S. Mende, Alignment and calibration of the ICON-FUV instrument: development of a vacuum UV facility. Proc. SPIE 9905, 99052W (2016c)

J.J. Makela, M.C. Kelley, A. González Sixto, N. Aponte, R.P. McCoy, Ionospheric topography maps using multiple-wavelength all-sky images. J. Geophys. Res. 106, 29161-29174 (2001). doi:10.1029/ 2000JA000449

L. Mandel, Image fluctuations in cascade intensifiers. Br. J. Appl. Phys. 10, 233-234 (1959)

R.R. Meier, D.E. Anderson, Determination of atmospheric composition and temperature from the UV airglow. Planet. Space Sci. 31, 967-976 (1983). doi:10.1016/0032-0633(83)90088-0

R.R. Meier, J.M. Picone, Retrieval of absolute thermospheric concentrations from the far UV dayglow: an application of discrete inverse theory. J. Geophys. Res. 99, 6307-6320 (1994). doi:10.1029/93JA02775

R.R. Meier et al., Remote sensing of Earth's limb by TIMED/GUVI: retrieval of thermospheric composition and temperature. Earth Space Sci. 2, 1-37 (2015). doi:10.1002/2014EA000035

S.B. Mende, Observing the magnetosphere through global auroral imaging, 2. observing techniques. J. Geophys. Res. Space Phys. 121, 10,638-10,660 (2016). doi:10.1002/2016JA022607

S.B. Mende, H. Heetderks, H.U. Frey, M. Lampton, S.P. Geller, S. Habraken, E. Renotte, C. Jamar, P. Rochus, J. Spann, S.A. Fuselier, J.-C. Gerard, R. Gladstone, S. Murphree, L. Cogger, Far ultraviolet imaging from the IMAGE spacecraft, 1: system design. Space Sci. Rev. 91, 243-270 (2000a)

S.B. Mende, H. Heetderks, H.U. Frey, M. Lampton, S.P. Geller, R. Abiad, O.H.W. Siegmund, A.S. Tremsin, J. Spann, H. Dougani, S.A. Fuselier, A.L. Magoncelli, M.B. Bumala, S. Murphree, T. Trondsen, Far ultraviolet imaging from the IMAGE spacecraft, 2: wideband FUV imaging. Space Sci. Rev. 91, 271285 (2000b)

S.B. Mende, H. Heetderks, H.U. Frey, J.M. Stock, M. Lampton, S.P. Geller, R. Abiad, O.H.W. Siegmund, S. Habraken, E. Renotte, C. Jamar, P. Rochus, J.-C. Gerard, R. Sigler, H. Lauche, Far ultraviolet imaging from the IMAGE spacecraft, 3: spectral imaging of Lyman $-\alpha$ and OI $135.6 \mathrm{~nm}$. Space Sci. Rev. 91, 287-318 (2000c)

J.S. Murphree, R.A. King, T. Payne, K. Smith, D. Reid, J. Adema, B. Gordon, R. Wlochowicz, The Freja ultraviolet imager. Space Sci. Rev. 70, 421-446 (1994)

J. Qin, F. Kamalabadi, J.J. Makela, Quantifying the inversion accuracy of simplified physical models for the nighttime OI 135.6 nm emission. J. Geophys. Res. Space Phys. 121, 5805-5814 (2016). doi:10.1002/ 2016JA022720

M.A. Quijada, S. Rice, E. Mentzell, Enhanced MgF2 and LiF over-coated al mirrors for FUV space astronomy, in Modern Technologies in Space- and Ground-Based Telescopes and Instrumentation II. Proc. SPIE, vol. 8450 (2012), p. article 84502H, pp10. doi:10.1117/12.925579 
E. Sagawa, T. Maruyama, T.J. Immel, H.U. Frey, S.B. Mende, Global view of the nighttime low-latitude ionosphere by the IMAGE/FUV $135.6 \mathrm{~nm}$ observations. Geophys. Res. Lett. 30(10), 1534 (2003). doi:10.1029/2003GL017140

A.W. Stephan, R.R. Meier, S.L. England, H.U. Frey, S.B. Mende, T.J. Immel, Daytime O/N2 retrieval algorithm for the ionospheric connection explorer (ICON) (2017)

D.J. Strickland, J.S. Evans, L.J. Paxton, Satellite remote sensing of thermospheric O/N2 and solar EUV, 1: theory. J. Geophys. Res. 100, 12217-12226 (1995). doi:10.1029/95JA00574

M.R. Torr, D.G. Torr, M. Zukic, R.B. Johnson, J. Ajello, P. Banks, K. Clark, K. Cole, C. Keffer, G. Parks, B. Tsuratani, J. Spann, A far ultraviolet imager for the international solar-terrestrial physics mission. Space Sci. Rev. 71, 329 (1995)

C.W. Wilkins, S.B. Mende, H.U. Frey, S.L. England, Time-delay integration imaging with ICON's farultraviolet spectrograph. Space Sci. Rev. (2017) 